DOI: https://doi.org/10.15517/rce.v37i2.40127

\title{
COSTA RICA: ESTIMACIONES DE LA TASA DE DESEMPLEO QUE NO ACELERA LA INFLACIÓN
}

\author{
COSTA RICA: ESTIMATING THE NON-ACCELERATING INFLATION RATE OF \\ UNEMPLOYMENT
}

\author{
Cristian Álvarez Corrales ${ }^{1}$ \\ Evelyn Muñoz-Salas ${ }^{2}$
}

Recibido: 24/05/2019

ABSTRACT Aprobado: 02/12/2019

In this paper we present estimations of the Non-accelerating Inflation Rate of Unemployment or NAIRU for the Costa Rican economy. We highlight the following results: The NAIRU displays an upward trend since the early 90 s which was accentuated after the Great Recession when the Costa Rican inflation rate exhibits historically low levels; the estimations of the unemployment gap (the difference between the observed unemployment rate and the NAIRU) indicates that this gap was negative in the nineties and the first half of 2000's but became positive afterwards. At the second quarter of 2017, the different estimates of the unemployment gap coincide in pointing out that this gap was closed, indicating the absence of aggregate demand pressures. In addition, since 2004, there has been a strengthening in the relationship between inflation and the unemployment gap.

KEY WORDS: NAIRU, UNEMPLOYMENT, INFLATION, PHILLIPS CURVE, AGGREGATE DEMAND.. JEL CLASIFICATION JEL: E24, E31, E52.

\section{RESUMEN}

Este documento presenta varias estimaciones de la tasa de desempleo que no acelera la inflación (NAIRU por sus siglas en inglés) para Costa Rica. De los resultados obtenidos, destaca lo siguiente: este indicador muestra una tendencia creciente desde inicios de los noventa, que se acentúa posterior a la Gran Recesión, periodo a partir del cual Costa Rica exhibe tasas de inflación históricamente bajas; las estimaciones de brecha de desempleo (diferencia entre la tasa de desempleo observada y la NAIRU) apuntan a que desde inicios de los noventa $y$ hasta los primeros años de la década de los 2000, esta tiende a ser mayormente negativa, $y$ positiva en adelante. Para el segundo trimestre de 2017, las diferentes estimaciones coinciden en señalar que la brecha de desempleo estaba cerrada, indicando la ausencia de presiones de demanda agregada. Asimismo, a partir del año 2004 se encuentra un fortalecimiento en la relación entre brecha de desempleo e inflación.

PALABRAS CLAVE: NAIRU, DESEMPLEO, INFLACIÓN, CURVA DE PHILLIPS, DEMANDA AGREGADA.

CLASIFICACIÓN JEL: E24, E31, E52.

1 Banco Central de Costa Rica, Departamento de Investigación Económica; Código Postal 10058-1000; San José Costa Rica; christalvarez@hotmail.com

2 Banco Central de Costa Rica, Departamento de Investigación Económica; Código Postal 10058-1000; San José Costa Rica; munozse@bccr.fi.cr 


\section{INTRODUCCIÓN}

En este trabajo se estima la $\mathrm{NAIRU}^{3}$ o tasa de desempleo que no acelera la inflación, para Costa Rica. Obtener una medida de la brecha de desempleo (la diferencia entre la tasa de desempleo observada y la NAIRU) es útil por cuanto este indicador provee información complementaria acerca de las presiones de demanda que se pueden estar generando en la economía y, por lo tanto, puede servir como insumo para proyectar la inflación en el corto y mediano plazo, así como para la toma de decisiones de política monetaria. Para el caso de Costa Rica, Segura y Vindas (2012) realizan una estimación de la NAIRU de forma simultánea con otras variables. No obstante, el objetivo primordial de su estudio no es estimar la NAIRU, sino la tasa de interés real neutral. Por lo tanto, esta investigación constituye una primera aproximación al estudio de la NAIRU como motivación principal.

Dado que la NAIRU no es una variable observable, debe ser estimada con base en algún método econométrico. En este trabajo se estima con base en una variedad de métodos para el periodo comprendido entre el primer trimestre de 1991 y el segundo de 2017. En primer lugar, se obtiene una estimación de una NAIRU constante, a partir de la estimación de una Curva de Phillips. Luego esta misma ecuación se estima de manera recursiva para obtener una NAIRU que varía en el tiempo. Asimismo, con el fin de brindar robustez a la estimación de una NAIRU que cambia en el tiempo, se recurre al método sugerido por Ball y Mankiw (2002), el filtro de Hodrick-Prescott (HP) y el filtro de Kalman.

De la estimación de una NAIRU constante, se tiene que para Costa Rica ésta se ubica en 8,7\%. Sin embargo, el intervalo de confianza al 95\% estimado con base en el método propuesto por Staiger, Stock y Watson (1996) es sumamente amplio, lo cual sugiere que la NAIRU se ubica en un rango comprendido entre 3,9 y 13,1\%. En lo que respecta a las mediciones que varían en el tiempo, coinciden en evidenciar una tendencia creciente de este indicador en Costa Rica, en consonancia con lo evidenciado por la tasa de desempleo, la cual parece haber experimentado un quiebre estructural luego de la crisis financiera internacional, pasando a mostrar tasas sustancialmente mayores a las exhibidas previo a esta crisis. A excepción de la estimación de la NAIRU variable con el filtro HP, las brechas de desempleo obtenidas (diferencia entre la tasa de desempleo observada y la NAIRU) con los otros métodos muestran una relación negativa con la tasa de inflación.

Un aspecto que se estudia en esta investigación tiene que ver con la evolución de la relación negativa que cabe esperar entre desempleo (o su brecha) y la tasa de inflación. Durante la reciente Gran Recesión, la inflación en Estados Unidos cayó mucho menos que lo pronosticado por la Curva de Phillips. Asimismo, los bajos niveles de desempleo experimentados en este país durante los últimos años (incluso por debajo de la NAIRU) no han estado acompañados de un incremento significativo en la tasa de inflación. Por lo tanto, estos hechos contradictorios han permitido a los economistas cuestionar si esta relación se ha debilitado o incluso desaparecido.

Con la intención de investigar si la relación entre brecha de desempleo e inflación continúa siendo válida en Costa Rica, se realiza un análisis recursivo para determinar la forma en que la relación entre estas dos variables ha evolucionado en el tiempo. Se estima una ecuación de Curva de Phillips donde las presiones sobre la demanda agregada se aproximan con las cinco brechas de desempleo estimadas. El resultado de este análisis muestra que, a partir del año 2004, se observa un fortalecimiento de la relación entre inflación y brecha de desempleo, en tanto el coeficiente asociado con el efecto de la brecha de desempleo sobre la inflación tiende a ser más negativo y, en algunos casos, presenta una mayor significancia estadística. Solo las brechas estimadas con la NAIRU constante $y$ la que se obtiene con el filtro de Kalman presentan evidencia de causar en sentido de Granger a la inflación, por lo que podrían usarse para efectos de elaborar pronósticos de la inflación.

El término NAIRU es un acrónimo para Non Accelerating Inflation Rate of Unemployment. 
El documento está organizado de la siguiente manera. La sección II aborda los principales conceptos asociados a la NAIRU y proporciona una revisión de la literatura relacionada con esta variable, adicionalmente provee una descripción de la relación entre la inflación y el desempleo en Costa Rica durante el periodo 1991-2017. La sección III describe los datos utilizados para aproximar la NAIRU. Las metodologías de estimación y los respectivos resultados se discuten en la sección IV. Finalmente, la sección V proporciona las principales conclusiones.

\section{REVISIÓN DE LITERATURA}

\section{Aspectos conceptuales}

El concepto de la NAIRU fue introducido por Modigliani y Papademos (1975) y parte del reconocimiento de que la sociedad enfrenta en el corto plazo una disyuntiva entre desempleo e inflación. Como sostienen Ball y Mankiw (2002), la política monetaria y en general las presiones de demanda pueden ubicar la tendencia de la inflación y el desempleo en direcciones opuestas en el corto plazo. De esta forma, cuando el desempleo se ubica por debajo de la NAIRU, existirán presiones inflacionarias. Mientras que, si ocurre lo contrario, entonces, habrá presiones para que la inflación se reduzca. Por lo tanto, implícito en este análisis está el hecho de que debe existir una tasa de desempleo en la cual la inflación se mantiene constante ${ }^{4}$. En el largo plazo, sin embargo, el uso de la política monetaria para incentivar la demanda agregada no puede generar mayores reducciones en la tasa de desempleo sin provocar una tasa de inflación progresivamente mayor. Por lo tanto, es reconocido que en el largo plazo tal disyuntiva entre desempleo e inflación no existe, por lo que la tasa de desempleo depende de factores estructurales, mientras que la inflación es un fenómeno monetario.

Por otra parte, un concepto relacionado y a veces confundido con la NAIRU es la tasa natural de desempleo, el cual fue propuesto por Milton Friedman en su discurso ante la Asociación Americana de Economía en 1968. No obstante, ambos conceptos no son sinónimos. A diferencia de la NAIRU, la tasa natural está relacionada con el equilibrio del mercado de trabajo, los factores microeconómicos que determinan su estructura, así como con las decisiones de las empresas y los hogares que afectan la demanda y la oferta de trabajo. Estrella y Mishkin (1998) definen la tasa natural de desempleo como la tasa que se observaría una vez que los factores cíclicos de corto plazo se hayan manifestado; mientras que, para estos autores, la NAIRU se debería interpretar como la tasa de desempleo congruente con una inflación estable en el corto plazo. Así, por ejemplo, un choque oferta que incremente la inflación requerirá de una reducción en la NAIRU para contrarrestar el efecto inflacionario de este choque.

En lo que respecta a la definición de la NAIRU, Boone et al. (2001) plantean tres posibilidades de acuerdo con el horizonte que considera cada una. La NAIRU de corto plazo, se define como la tasa de desempleo necesaria para estabilizar la inflación en su nivel actual en el siguiente periodo. Esta definición implica que debe ser muy volátil, ya que requiere que la NAIRU varíe en cada periodo en una magnitud suficiente como para compensar cualquier choque que incremente la inflación. Por su parte, la NAIRU de mediano plazo es el nivel de equilibrio al que la tasa de desempleo converge cuando no ocurren choques de oferta $y$ la inflación ha completado su proceso de ajuste ante choques previos. Finalmente, la NAIRU de largo plazo corresponde a la tasa de desempleo en el estado estacionario, esto es, cuando se ha ajustado totalmente a todos los choques de oferta y efectos de política incluyendo aquellos con efectos de larga duración.

4 Implícito también está el supuesto de que debe existir una relación entre el desempleo y la inflación de salarios, los cuales son en última instancia, los que ejercen presión sobre la inflación. 
De estas tres definiciones, solamente la NAIRU de corto y mediano plazo suelen estimarse. La de corto plazo puede medirse directamente, mientras que la de mediano plazo puede calcularse controlando por los factores que afectan la inflación en el corto plazo. Por lo tanto, el concepto relevante para esta investigación es la NAIRU de mediano plazo. De acuerdo con Boone et al. (2001), la NAIRU de largo plazo es más difícil de estimar debido a la dificultad de cuantificar las influencias individuales con efectos de larga duración.

\section{Métodos de estimación}

Respecto de la estimación de la NAIRU, Ball y Mankiw (2002) plantean dos obstáculos. El primero asociado con la dificultad de hacer una correcta estimación econométrica de la ecuación de Curva de Phillips que, por lo general, se utiliza para estimar la NAIRU, lo cual llevaría a obtener estimaciones inconsistentes de los parámetros. Esto, por cuanto es de esperar que los choques de oferta estén, de forma contemporánea, correlacionados con la NAIRU. La forma en que la literatura ha lidiado con este problema básicamente consiste en suponer que los choques de oferta y la NAIRU no están correlacionados de forma contemporánea.

El otro problema relacionado con la estimación consiste en la obtención de intervalos de confianza. Staiger, Stock y Watson (1996) encontraron que la NAIRU en 1990 se ubicaba en 6,2\% en Estados Unidos. El intervalo de confianza al 95\% asociado con esta estimación indicaba que esta variable se ubicaba en un rango entre 5,1 y 7,7\%. Los autores mencionan que este intervalo de confianza tan amplio puede deberse a la incertidumbre acerca de los parámetros del modelo, el hecho de que la NAIRU es aleatoria y la incertidumbre relacionada con la especificación del modelo dado que no se conoce el modelo "correcto".

Otra dificultad práctica que plantean Szeto y Guy (2004) está relacionada con el uso de las expectativas de inflación. Una opción consiste en utilizar las expectativas de inflación provenientes de una encuesta. Por otro lado, también se ha hecho el supuesto de que las expectativas son adaptativas, por lo que la inflación esperada se supone igual a la del periodo anterior. Dado que la serie de expectativas de inflación disponible para Costa Rica empieza a partir del año 2006, en esta investigación se decidió usar el supuesto de expectativas adaptativas en las diferentes estimaciones de la Curva de Phillips.

Respecto a la estimación de la NAIRU, tradicionalmente se ha recurrido una variedad de métodos entre los que destacan filtros estadísticos, métodos estructurales y ecuaciones de forma reducida ${ }^{5}$. En cuanto a los filtros estadísticos, estos descomponen la serie de desempleo en una tendencia y un componente cíclico, ignorando la relación que pueda existir entre la inflación y el desempleo. La serie de tendencia que resulta de esta descomposición es considerada como una estimación de la NAIRU. El principal supuesto detrás de estos métodos es que, dada la ausencia de una relación de largo plazo entre inflación y desempleo, entonces en promedio, la tasa de desempleo debe fluctuar alrededor de la NAIRU si las fuerzas que equilibran la economía son los suficientemente fuertes como para traer de vuelta la tasa desempleo de regreso a su tendencia.

Por su parte, los modelos estructurales calculan la NAIRU como el resultado del equilibrio en un modelo de fijación de precios y salarios (Fabiani \& Mestr, 2000; Richardson et al., 2000). Sin embargo, dado que la tasa de desempleo estimada de esta forma supone un ajuste total del comportamiento de los trabajadores y los empresarios a todos los choques, la tasa de desempleo de equilibrio está más relacionada con el concepto de tasa natural discutido anteriormente que con el de la NAIRU.

5 Una descripción más detallada de los métodos aquí reseñados puede encontrarse en Boone et al. (2001). 
El cómputo de la NAIRU mediante la estimación de ecuaciones de forma reducida es, por mucho, el método más popular para estimar esta variable. La principal ventaja es que permite tomar en cuenta directamente la relación entre la inflación y el desempleo, y controlar por otros factores que, en el corto plazo, afectan la inflación, como los choques de oferta y las expectativas de inflación. Como la mayor parte de las especificaciones utilizadas incluyen estos tres elementos, al modelo citado se le conoce también como modelo triangular o Curva de Phillips aumentada con choques de oferta (Gordon, 1997). Con este enfoque, la NAIRU puede estimarse mediante una simple regresión de mínimos cuadrados ordinarios (MCO), con el supuesto de que la NAIRU es constante.

Otro método de estimación común bajo este enfoque ha sido el filtro de Kalman. Una ventaja de este método sobre la estimación MCO es que permite obtener una NAIRU que varía en el tiempo, así como una medida de la incertidumbre asociada con su estimación. Asimismo, permite estimar la Curva de Phillips simultáneamente con la NAIRU. Este es un enfoque similar al de Laubach y Williams (2003) y utilizado por Segura y Vindas (2012) para Costa Rica. En este caso, la NAIRU se estima de forma conjunta con la tasa de interés real neutral en el contexto de la estimación de un modelo semiestructural resuelto con el filtro de Kalman. Estos autores obtienen una estimación de la NAIRU para el período 2001-2011 que exhibe una evolución bastante suave, similar en apariencia a la que se podría obtener con el filtro HP. Asimismo, a juzgar por una banda de confianza de un error estándar, esta parece estimarse con bastante precisión. La NAIRU estimada oscila entre el 5 y el 8\%, y su valor promedio se ubica en torno al $6 \%{ }^{6}$.

\section{Evolución reciente de la relación entre inflación y desempleo}

Un fenómeno que ha llamado la atención de los economistas en años recientes tiene que ver con el denominado episodio de "desinflación no observada" 7 al cual se han referido economistas como Krugman (2015), Kiley (2015) y Blanchard (2016). En particular, estos autores sugieren que los niveles de inflación observados en los Estados Unidos durante la Gran Recesión fueron sorpresivamente altos. Con base en una relación de Curva de Phillips se hubiera esperado que, en la fase recesiva, la inflación cayera mucho más de lo efectivamente observado, dados los altos niveles de desempleo experimentados. Asimismo, en los últimos años una tasa de desempleo por debajo de lo que se considera la NAIRU no ha conllevado un incremento sustancial de la inflación. Por lo tanto, esto ha motivado a que algunos economistas se planteen si la relación entre inflación y desempleo se ha debilitado o, si incluso, ha desaparecido por completo.

Se han brindado algunas explicaciones para este fenómeno, principalmente relacionadas con la forma en la que las expectativas de inflación y la brecha de desempleo afectan la inflación en la Curva de Phillips. Por ejemplo, algunos autores como Ball y Mazumder (2011), Stock (2011) y Coibion y Gorodnichenko (2015) plantean que, conforme las expectativas de inflación se encuentran más ancladas en los Estados Unidos, el efecto de la brecha de desempleo sobre la inflación se torna más pequeño. Kiley (2015) encuentra que la relación entre las expectativas de inflación y la inflación pasada se ha debilitado sustancialmente desde mediados de la década de los noventa. Por su parte, Blanchard (2016) muestra que la inflación ha pasado a depender, en mayor medida, de las expectativas de inflación y, en menor proporción, de la inflación pasada, ello limita el efecto de la brecha de desempleo sobre la inflación.

Otra explicación se relaciona con el posible aplanamiento de la Curva de Phillips. Ball y Mazumder (2011) sugieren que las rigideces nominales en precios y salarios aumentan en un ambiente de inflación baja, por lo que una mayor parte del ajuste en la demanda agregada recae sobre el producto en vez de la inflación. Además, también se ha encontrado una menor respuesta 
de la inflación a la debilidad del mercado laboral en un ambiente de inflación baja (Daly \& Hobjin, 2014). En cuanto a la evidencia empírica de este aplanamiento en la Curva de Phillips, Blanchard, Cerutti y Summers (2015) analizan una muestra de 23 países desarrollados durante 50 años y encuentran que el efecto de la brecha de desempleo sobre la inflación ha disminuido desde los años setenta. Sin embargo, la mayor parte de la reducción se dio con anterioridad a la década de los noventa, mientras que el coeficiente se ha mantenido estable desde entonces y significativo en muchos casos. Por lo tanto, no parece haber evidencia de que la relación haya cambiado luego de la Gran Recesión.

Algunos economistas han planteado que uno de los factores que puede explicar la modesta caída de la inflación durante la Gran Recesión se relaciona con que la tasa de desempleo en este período puede no aproximar bien el grado de debilidad de la economía. En este sentido, el gran y persistente incremento del desempleo de larga duración implica la posibilidad de que los desempleados se hayan desalentado y desconectado del mercado laboral en una magnitud suficiente como para dejar de ejercer una mayor presión a la baja de los salarios. La evidencia relacionada con esta explicación no es concluyente en el estudio de Ball y Mazumder (2011), mientras que Kiley (2015) y Cheung, Leung, y Lo (2017) no encontraron evidencia de que la tasa de desempleo de corto plazo fuera un mejor indicador de la debilidad del mercado laboral que la tasa de desempleo total.

\section{Desempleo e inflación en Costa Rica}

Este apartado provee una breve descripción de la relación entre la tasa de inflación y la tasa de desempleo en Costa Rica durante en el período 1991 a 2017. Los gráficos de la figura 1 muestran la evolución de estas dos variables en este lapso. Como puede verse, a lo largo de este periodo, ambas series muestran tendencias opuestas.

La tasa de inflación muestra una tendencia a reducirse de forma gradual. En la década de los noventa, exhibe una alta volatilidad, con una amplitud de cerca de 20 p.p. y un promedio de $16,6 \%$. En la década siguiente, tanto su nivel como volatilidad, se reducen sensiblemente, la inflación promedio pasa a ser del 11\%. Asimismo, parece exhibir un quiebre estructural en el año 2009, de forma coincidente con el periodo asociado con la Gran Recesión, en tanto la inflación pasa a experimentar niveles mucho menores a los de años previos a esta crisis ${ }^{8}$.

A partir de ese año, la inflación en Costa Rica muestra niveles de un dígito. Entre 2010 y 2014, se mantuvo en un promedio del 5\%; luego del 2015, se reduce de forma pronunciada, principalmente por el choque favorable de precios de materias primas, en ese año y parte de 2016 mostró niveles negativos. Además, ha estado por debajo de la meta de inflación establecida por el BCCR para esos años.

8 Torres (2012) identifica un quiebre estructural en la tasa de inflación en Costa Rica en mayo de 2009 con base en la prueba de Bai y Perron (2003). 


\section{FIGURA 1 \\ INFLACIÓN Y DESEMPLEO EN COSTA RICA}
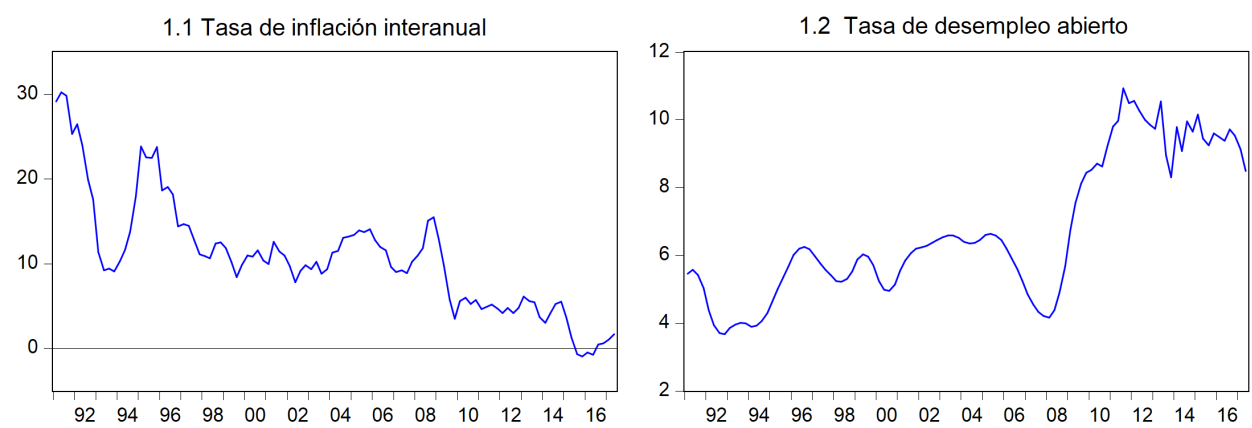

Fuente: Elaboración propia

Por su parte, la tasa de desempleo abierto se mostró relativamente estable entre los años 1991 y 2008 , con fluctuaciones alrededor de un promedio del 5,4\%, aunque con una leve tendencia al incremento. No obstante, a partir del año 2008 muestra un fuerte aumento, evidencia de un posible quiebre estructural en esta variable, el cual puede asociarse con los efectos de la crisis financiera internacional. A pesar de que el desempleo parece haberse reducido desde su pico de $10,9 \%$ en el tercer trimestre del 2011, se observa que este no ha retornado al nivel promedio que mostraba antes de la crisis, todo lo cual apunta a que se puede estar en presencia de un fenómeno de histéresis en la tasa de desempleo en Costa Rica9.

La tendencia que se observa en ambas series, referente a menores tasas de inflación acompañadas de mayores tasas de desempleo sugiere una posible relación negativa entre ambas, tal y como lo indica la Curva de Phillips. Lo anterior es confirmado por la figura 2, la cual presenta gráficos de dispersión para mostrar la relación entre inflación y desempleo en Costa Rica, durante la muestra total y varias submuestras. En cada gráfico se incluye una línea de tendencia junto con la ecuación que la estima y el R cuadrado.

9 Both in Krugman (1980) and in Melitz (2003) the economies of scale are introduced by simply having fixed costs of production and a constant marginal cost. 
FIGURA 2

RELACIÓN ENTRE INFLACIÓN Y DESEMPLEO EN COSTA RICA*
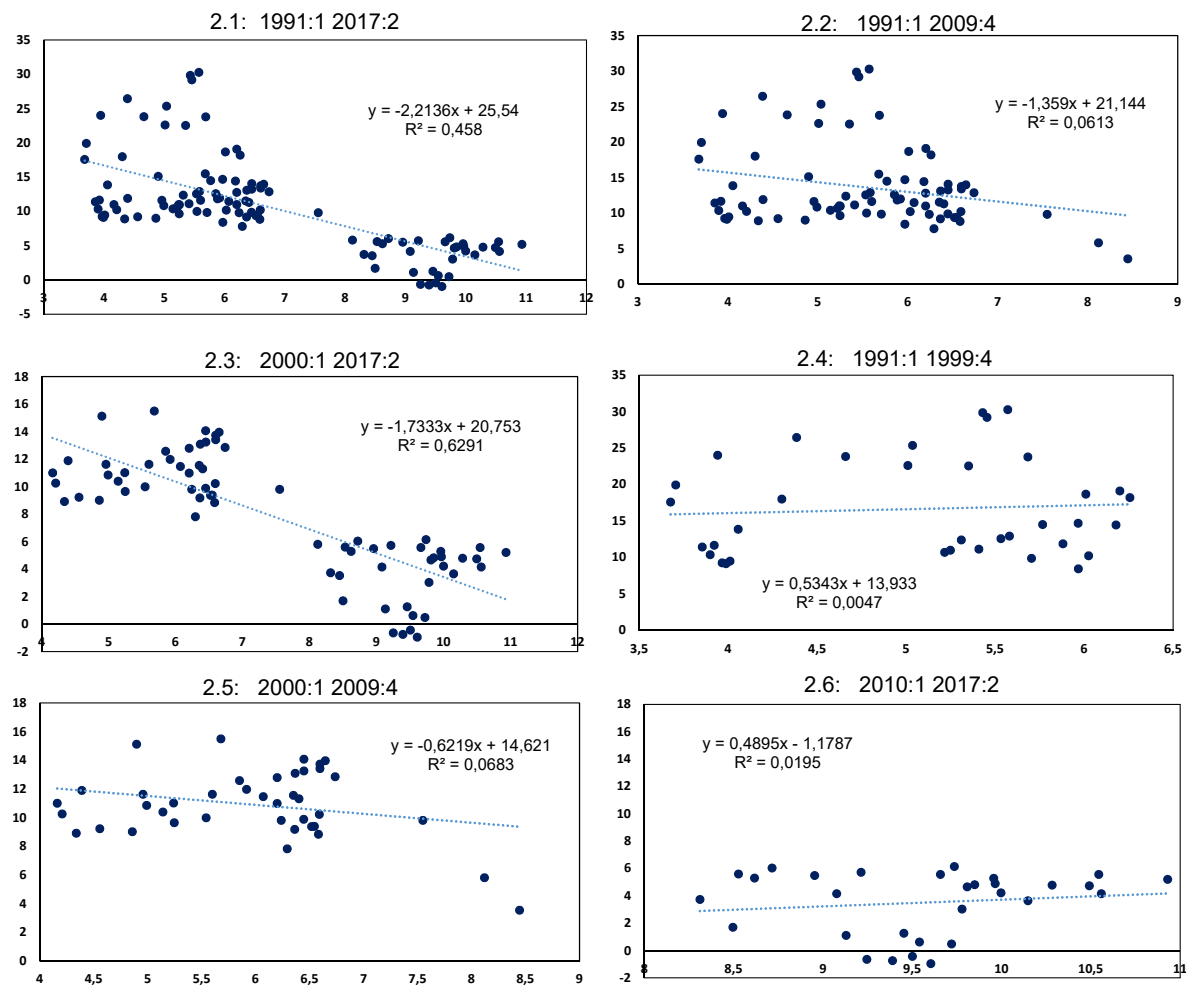

* Inflación en eje izquierdo y desempleo en eje derecho.

Fuente: Elaboración propia.

Como se puede apreciar, cuando se analiza la muestra total, la relación entre inflación y desempleo es negativa. También se presenta esta relación para dos submuestras: 1991-2009 y 2000 2017, aunque con diferente intensidad. Cuando se analiza las primeras dos décadas esta relación es más débil, la línea de tendencia muestra una inclinación menor, dando a su vez como resultado un coeficiente más pequeño. Por su parte, cuando se analizan los años transcurridos del actual milenio, la relación continúa siendo negativa.

Los gráficos 2.4, 2.5 y 2.6 muestran la relación entre inflación y desempleo para cada una de las décadas comprendidas en este estudio. Como se concluye de estos gráficos, la relación negativa entre inflación y desempleo no se ha manifestado en todas estas décadas. Durante los noventa y los últimos siete años de la muestra, la relación entre ambas series fue ligeramente positiva. En todos los casos, la magnitud de la asociación entre desempleo e inflación ha sido menor (en valor absoluto) que la aproximada por el valor de los coeficientes en los gráficos 2.1, 2.2 y 2.3. No obstante, lo que se puede inferir de estos gráficos es que, conforme se incorpora información más reciente, esta relación parece debilitarse, observándose que si se toma en consideración los datos a partir del año 2010 la relación es más bien positiva. 
Una de las explicaciones que se ha brindado para la ruptura de la relación entre inflación $y$ desempleo es que los salarios pueden reaccionar en menor medida a los niveles de desempleo cuando la inflación es baja. Como se mencionó previamente, la inflación en Costa Rica experimentó un cambio estructural en el año 2009 y muestra niveles de inflación sustancialmente menores a los exhibidos en décadas anteriores y a partir de entonces, esta ha mostrado niveles de un dígito. La siguiente sección proporciona un análisis cuantitativo de evolución de la relación entre inflación y desempleo en Costa Rica.

\section{DATOS}

Uno de los elementos que complica la estimación de la NAIRU para la economía costarricense es la ausencia de una serie de desempleo recopilada con una misma metodología y periodicidad para un período suficientemente extenso. Para el período comprendido entre 1991 y el año 2009, se tiene una serie de la tasa de desempleo abierto ${ }^{10}$ con periodicidad anual, cuya fuente es la Encuesta de Hogares de Propósitos Múltiples (EHPM) realizada por el Instituto Nacional de Estadística y Censos (INEC).

A partir del año 2010, el INEC sustituye la EHPM por la Encuesta Nacional de Hogares (ENAHO) también de periodicidad anual, $y$ de forma coincidente, a partir del segundo trimestre de ese año esta Entidad empieza a obtener estadísticas de empleo con una mayor cobertura de variables y una mayor periodicidad a partir de la Encuesta Continua de Empleo (ECE). Esta encuesta, a diferencia de la EHPM y la ENAHO, realiza un cálculo trimestral de la tasa de desempleo abierto.

Una consideración importante es que la medición de la fuerza de trabajo que se lleva a cabo en la ECE contabiliza a las personas mayores de 15 años como parte de la fuerza laboral, mientras que las otras dos encuestas las incluyen a partir de los 12 años. Por tal motivo, las series de desempleo obtenidas de la EHPM y la ENAHO no son directa y estrictamente comparables con el dato que resulta de la ECE. Sin embargo, para efectos de investigación, el INEC facilitó una serie de desempleo abierto que corrige por esta discrepancia para el periodo 1991-2010, por lo que la serie de desempleo abierto usada en esta investigación se calcula con base en una fuerza de trabajo conformada por población mayor de 15 años. Esta serie es trimestralizada siguiendo el método de Denton (1971), para obtener una serie con periodicidad trimestral para este lapso.

En las diferentes estimaciones se utiliza la tasa de inflación interanual calculada con base en el Índice de precios al consumidor (IPC) calculado por el INEC. Asimismo, se hace uso de un indicador de inflación subyacente como el indicador de inflación de media truncada (IMT) ${ }^{11}$. Además, para capturar el efecto de los choques de oferta, se utiliza la inflación promedio de los principales socios comerciales de Costa Rica, la cual pondera las inflaciones de estos socios de acuerdo con su importancia relativa en el comercio con Costa Rica ${ }^{12}$. El periodo analizado abarca desde el primer trimestre de 1991 hasta el segundo trimestre de 2017. En el caso de la IMT, se tienen datos a partir del primer trimestre de 1997, lo cual limita en alguna medida las estimaciones de la NAIRU que se obtengan del uso de este indicador.

10 La tasa de desempleo abierto corresponde a la fracción de la fuerza de trabajo que no cuenta con un empleo y que se encuentra en disposición de trabajar.

11 La IMT excluye del cálculo de la inflación de cada mes, aquellos productos del IPC cuyas variaciones de precio son las más bajas y las más altas. La distribución de cambios de precio es truncada de forma asimétrica, eliminando el $30 \%$ de cambios de precios a la izquierda del percentil de la media (percentil 60) y el 10\% a la derecha. El porcentaje de truncamiento se mantiene fijo a lo largo del tiempo y el conjunto de productos que se descarta cada mes no es fijo.

12 También se probó inicialmente controlar por el efecto traspaso del tipo de cambio a la inflación; sin embargo, no resultó estadísticamente significativo en la especificación de Curva de Phillips. 
Un paso previo al análisis de regresión consiste en obtener conclusiones acerca de las propiedades de estacionariedad de las series utilizadas, de modo que las inferencias estadísticas obtenidas sean válidas estadísticamente. El cuadro 1 brinda los resultados de las pruebas de raíz unitaria para estas series. Se aplicaron las pruebas ${ }^{13}$ ADF, PP, KPSS y la prueba ADF que controla por la presencia de cambios estructurales en las series. Esta prueba es relevante, puesto que como se aprecia en la figura 1, el comportamiento de las series de interés a lo largo del periodo de estudio, parecen mostrar un quiebre estructural. De igual forma, las pruebas se aplicaron para el modelo con constante y tendencia, dado que es el que mejor parece describir los datos.

CUADRO 1

PRUEBAS DE RAÍZ UNITARIA

\begin{tabular}{lcccccc} 
& \multicolumn{1}{c}{ ADF } & PP & KPSS & ADF BREAK C & ADF_BREAK_C \\
Inflación & $-5,54$ & $* * *$ & $-3,01$ & 0,08 & $-3,58$ & $-6,85$ \\
IMT & $-2,49$ & $-2,44$ & $0,19 * * *$ & $-4,68 * *$ & $-4,57$ \\
Inf.Socios & $-3,36 * *$ & $-2,75$ & 0,11 & $-3,74$ & $-4,71$ & $-4,94 *$ \\
Desempleo & $-3,12$ & $-2,52$ & 0,11 & $-4,22 *$ & $*$ \\
\hline
\end{tabular}

***,**, Significativo al 1\%, 5\% y 10\% de significancia, respectivamente.

a Quiebre estructural en la constante.

$b$ Quiebre estructural en la constante y la tendencia.

Fuente: Elaboración propia.

Como se puede ver, los resultados no son concluyentes respecto al orden de integración de las variables. La prueba ADF rechaza la hipótesis de raíz unitaria al $1 \%$ de significancia para la inflación y al 10\% para la inflación de los socios comerciales, mientras que no lo hace para la tasa de desempleo. La prueba PP no rechaza la existencia de raíces unitarias en todas las variables, mientras que la prueba KPSS más bien muestra lo contrario ${ }^{14}$, a excepción de la IMT, para la cual se rechaza la hipótesis de estacionariedad al $5 \%$ de significancia.

En lo que respecta a las pruebas que toman en cuenta la presencia de cambios estructurales, estas rechazan la hipótesis nula de raíz unitaria para la tasa de desempleo a una significancia del $10 \%$, lo que confirma las sospechas de que la presencia de un cambio estructural podría estar sesgando las pruebas AD y PP al no rechazo de la hipótesis nula de raíz unitaria. Por su parte, la tasa de inflación parece mostrar evidencia de cambio estructural en la constante y la tendencia, confirmando la hipótesis de estacionariedad. Respecto a la IMT, la prueba de raíz unitaria que permite quiebres estructurales en el intercepto sugiere que esta serie es estacionaria con cambio estructural a un nivel de significancia del 5\%. Para el caso de la inflación de los socios comerciales, las pruebas con cambio estructural continúan indicando que tiene raíz unitaria.

En resumen, parece que la evidencia es más fuerte en torno a que la tasa de inflación medida por el IPC parece ser estacionaria y la IMT estacionaria con evidencia de cambio estructural en la constante. Para la tasa de desempleo, parece haber indicios de un quiebre estructural tanto en la constante como la tendencia, aunque esta evidencia es menos sólida. Finalmente, la evidencia de estacionariedad en la inflación de los socios comerciales parece ser débil. A pesar de estos resultados, las estimaciones que se presentan en la siguiente sección utilizan estas variables en niveles en tanto permiten modelar la inflación de una forma más coherente.

13 Dickey-Fuller aumentada, Phillips-Perron y Kwiatkowski-Phillips-Schmidt-Shin.

14 Esta prueba tiene como hipótesis nula que la serie es estacionaria. 


\section{METODOLOGÍA DE ESTIMACIÓN Y RESULTADOS}

A continuación, se describen los métodos utilizados para estimar la NAIRU en Costa Rica y se presentan los resultados de estas estimaciones. Un primer punto de partida consiste en obtener una NAIRU constante a partir de la Curva de Phillips. Además, se calcula un intervalo de confianza de acuerdo con el método de Staiger, Stock y Watson (1996).

\section{NAIRU constante}

De la estimación de los parámetros de la Curva de Phillips, es posible obtener una medida de la NAIRU, al suponer que la tasa de desempleo y el término de error no están correlacionados contemporáneamente. Se estima la siguiente ecuación para el periodo comprendido entre el primer trimestre de 1992 y el segundo trimestre de 2017:

$$
\pi_{t}=\beta_{0}+\beta_{1} \pi_{t-1}+\beta_{2} U_{t-2}+\beta_{3} \pi_{t}^{*}+\varepsilon_{t}
$$

Donde $\pi_{t}$ es la tasa de inflación interanual, $U_{t-2}$ es la tasa de desempleo abierto rezagada dos trimestres, $\pi_{\mathrm{t}}{ }^{*}$ es la inflación de socios comerciales y $\varepsilon_{t}$ es el término de error. La ecuación (1) resultó ser la mejor especificación en términos de la significancia de los coeficientes luego de probar con especificaciones con diferentes órdenes de rezago. Una vez estimada la ecuación (1) la NAIRU se obtiene como: $U^{*}=-\hat{\beta}_{0} / \hat{\beta}_{2}$. El resultado de estimar la ecuación (1) se presenta en el cuadro 2 , donde la ecuación para la IMT incluye su segundo rezago, así como la variación interanual del tipo de cambio nominal. Respecto a las ecuaciones por estimar, se supone la existencia de homogeneidad dinámica, supuesto que parece coherente con los datos ${ }^{15}$.

CUADRO 2

ESTIMACIÓN DE LA CURVA DE PHILLIPS

\begin{tabular}{|c|c|c|c|c|}
\hline \multirow{2}{*}{ VARIABLE } & \multicolumn{2}{|c|}{ INFLACIÓN IPC } & \multicolumn{2}{|c|}{ IMT } \\
\hline & Coeficiente & Valor-P & Coeficiente & Valor-P \\
\hline $\mathrm{C}$ & 2,17 & 0,08 & 1,35 & 0,05 \\
\hline$\pi_{t-1}$ & 0,78 & 0,00 & 1,07 & 0,00 \\
\hline$\pi_{t-2}$ & & & $-0,28$ & 0,00 \\
\hline$\pi_{t}^{*}$ & 0,43 & 0,00 & 0,37 & 0,00 \\
\hline$e_{t}$ & & & 0,04 & 0,01 \\
\hline$U_{t-2}$ & $-0,25$ & 0,01 & $-0,15$ & 0,01 \\
\hline R2 ajustado & \multicolumn{2}{|c|}{0,92} & \multicolumn{2}{|c|}{0,98} \\
\hline $\begin{array}{l}\text { Durbin- } \\
\text { Watson }\end{array}$ & \multicolumn{2}{|c|}{1,27} & \multicolumn{2}{|c|}{1,91} \\
\hline Estadístico F & \multicolumn{2}{|c|}{0,00} & \multicolumn{2}{|c|}{0,00} \\
\hline
\end{tabular}

Fuente: Elaboración propia.

15 No se rechaza la hipótesis nula de que la suma de los coeficientes asociados a la inflación rezagada y la inflación de socios comerciales es igual a 1 para la ecuación de Curva de Phillips con el IPC. Para la Curva de Phillips estimada con la IMT tampoco se rechaza esta hipótesis al añadir la variación interanual del tipo de cambio nominal. 
La NAIRU así obtenida es 8,7\%. Al estimar una ecuación similar con la IMT para el período comprendido entre el tercer trimestre de 1997 y el segundo de 2017, se obtiene una NAIRU muy similar $(8,75 \%)$.

Como se discutió en la sección 2, uno de los problemas inherentes a la estimación de la NAIRU por medio de la Curva de Phillips es la dificultad de obtener una medida de la incertidumbre asociada con su estimación dado que esta es una función no lineal de los parámetros estimados $\left(\hat{\beta}_{0}\right.$ y $\left.\hat{\beta}_{2}\right)$. Por medio del método Gaussiano, Staiger, Stock y Watson (1996) desarrollaron un procedimiento simple que permite verificar la hipótesis de que un valor dado de la NAIRU, $U_{0}^{*}$, pertenece al intervalo de confianza de la NAIRU estimada. Si no se rechaza esta hipótesis, entonces $U_{0}^{*}$ pertenece al intervalo de confianza. Para aplicar este método, primero se obtiene la suma de residuos al cuadrado de la ecuación (1) o suma de cuadrados no restringida (SRS). Luego se estima la siguiente ecuación para diferentes valores de $U_{0}^{*}$ :

$$
\pi_{t}=\beta_{1} \pi_{t-1}+\beta_{2}\left(U_{t-2}-U_{l}^{*}\right)+\beta_{3} \pi_{t}^{*}+\varepsilon_{t}
$$

Para cada estimación de la ecuación (2) con base en cada valor supuesto de $U_{i}^{*}$, se toma la suma de residuos al cuadrado a la que se puede denominar suma de cuadrados restringida (SRR) y se procede a calcular el estadístico F:

$$
F=\frac{\left[S R R_{U_{o}^{i}}-S r S\right]}{S R S /(T-K)}
$$

La NAIRU supuesta en cada estimación $\left(\mathrm{U}_{-} \mathrm{i}^{\wedge *}\right)$ varía en 0,05 p.p. con valores que van desde cero hasta el $15 \%$. Los valores de $\mathrm{U}_{-} \mathrm{i}^{\wedge *}$ que generan un valor menor del estadístico $\mathrm{F}$ que el valor de este estadístico al 5\% de significancia $(3,94)$ quedan incluidos dentro del intervalo. En este caso, dicho intervalo va del 3,9\% al 13,1\%; es decir, una amplitud de 9,2 p.p ${ }^{16}$. Por lo tanto, parece que existe una gran imprecisión respecto al valor estimado de la NAIRU con este método.

El gráfico 3.1 de la figura 3 muestra la NAIRU estimada en la ecuación para la inflación IPC junto con la tasa de desempleo y el intervalo de confianza calculado. No obstante, este intervalo parece poco plausible en tanto la tasa de desempleo observada nunca alcanza siquiera el límite superior del intervalo, mientras que el límite inferior sugiere una brecha de desempleo positiva la mayor parte del tiempo, lo cual no es coherente con la dinámica observada de la inflación.

16 El intervalo obtenido para la NAIRU calculada con base en la IMT es todavía más amplio, ubicándose entre 0,1 y 11,1\%. Por tanto, no tiene mucho valor informativo. 


\section{FIGURA 3}

NAIRU CONSTANTE

3.1 NAIRU y tasa de desempleo abierto

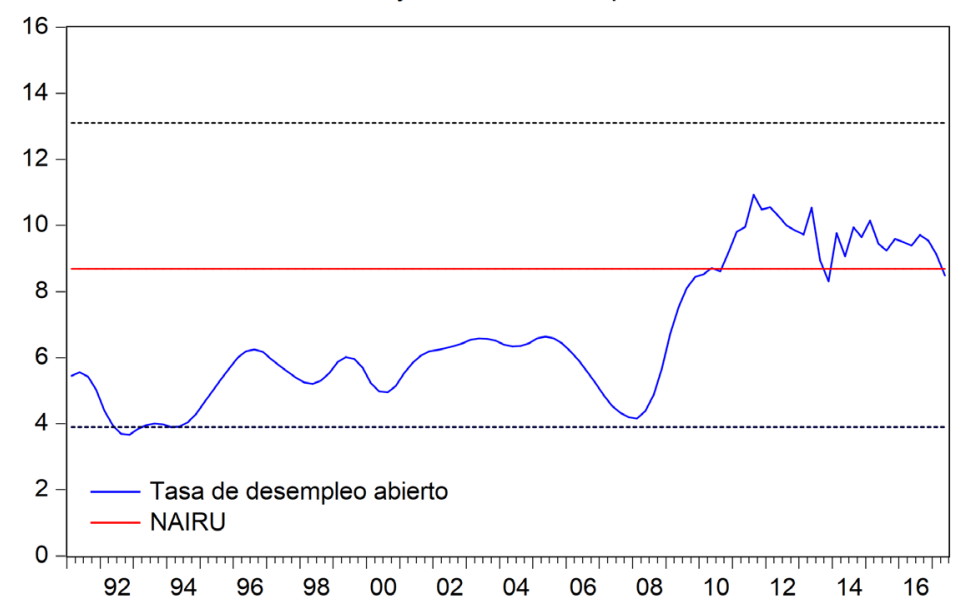

3.2 Inflación IPC y brecha de desempleo (NAIRU constante)

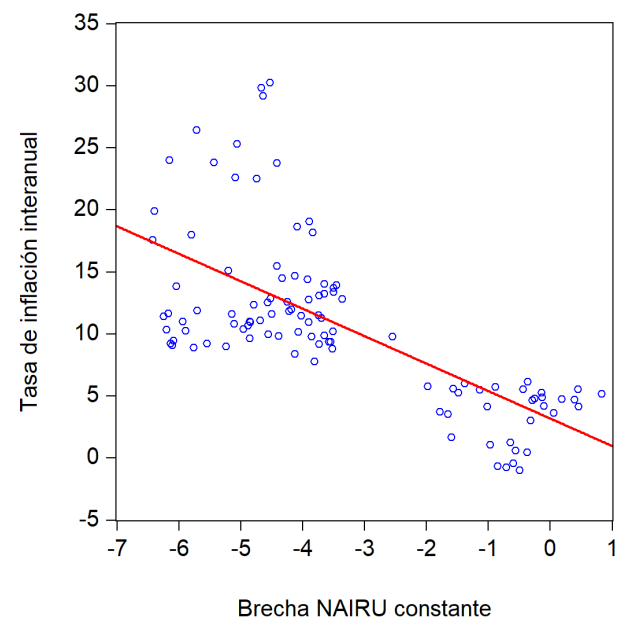

3.3 Inflación IMT y brecha de desempleo (NAIRU constante)

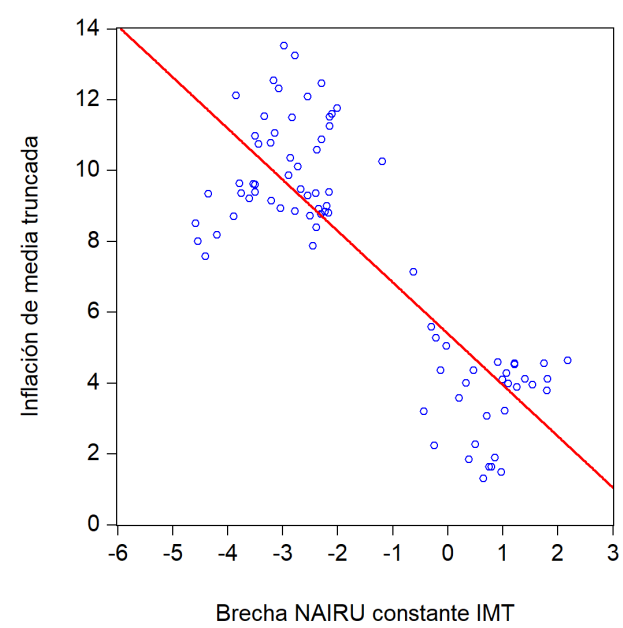

Fuente: Elaboración propia.

Como se puede observar, desde el año 1991 y hasta el 2010 la estimación puntual de la NAIRU indica que la brecha de desempleo era negativa. A partir de este último año la brecha de desempleo es más bien positiva la mayor parte del tiempo, lo cual pudo haber contribuido al proceso de desinflación que ha experimentado la economía costarricense durante esos años. Como se muestra en el gráfico, parece que al segundo trimestre del 2017 la brecha de desempleo se ha cerrado por lo que no existirían presiones de demanda por esta vía. Los gráficos 3.2 y 3.3 muestran que la brecha de desempleo calculada con ambas estimaciones de NAIRU están relacionadas de forma negativa con la inflación; la correlación entre ambas variables es de -0,68 en el caso de la inflación IPC y de $-0,83$ en el caso de la IMT. NAIRU recursiva 
Una opción para obtener una NAIRU que varíe en el tiempo es hacer una estimación recursiva de la ecuación (1) para una muestra cambiante. En este caso, se estima la ecuación (1) para una muestra inicial de 24 trimestres y se va añadiendo secuencialmente una observación al final de la muestra hasta agotar el total de observaciones. La NAIRU es calculada en cada de una de estas estimaciones de la misma forma que en el caso de la NAIRU constante, esto es, dividiendo el negativo del intercepto entre el coeficiente asociado a la tasa de desempleo ${ }^{17}$.

El panel izquierdo de la figura 4 muestra el resultado de la NAIRU estimada recursivamente. Luego de un breve periodo al inicio en que la NAIRU se reduce, esta muestra una tendencia creciente desde el año 2001 que se mantiene hasta el 2013, a partir de este año exhibe una leve tendencia a reducirse. Al igual que la NAIRU constante, esta estimación variable de la NAIRU muestra una brecha de desempleo negativa hasta el 2009, desde entonces ha pasado a ser positiva la mayor parte del tiempo.

Esta estimación variable también coincide con la NAIRU constante en evidenciar que la brecha de desempleo parece estar cerrada al segundo trimestre de 2017. La relación entre esta brecha de desempleo y la inflación que se muestra en el panel de la derecha de la figura 4 también es negativa. La correlación entre ambas es -0,74.

FIGURA 4

\section{NAIRU VARIABLE ESTIMADA DE FORMA RECURSIVA}
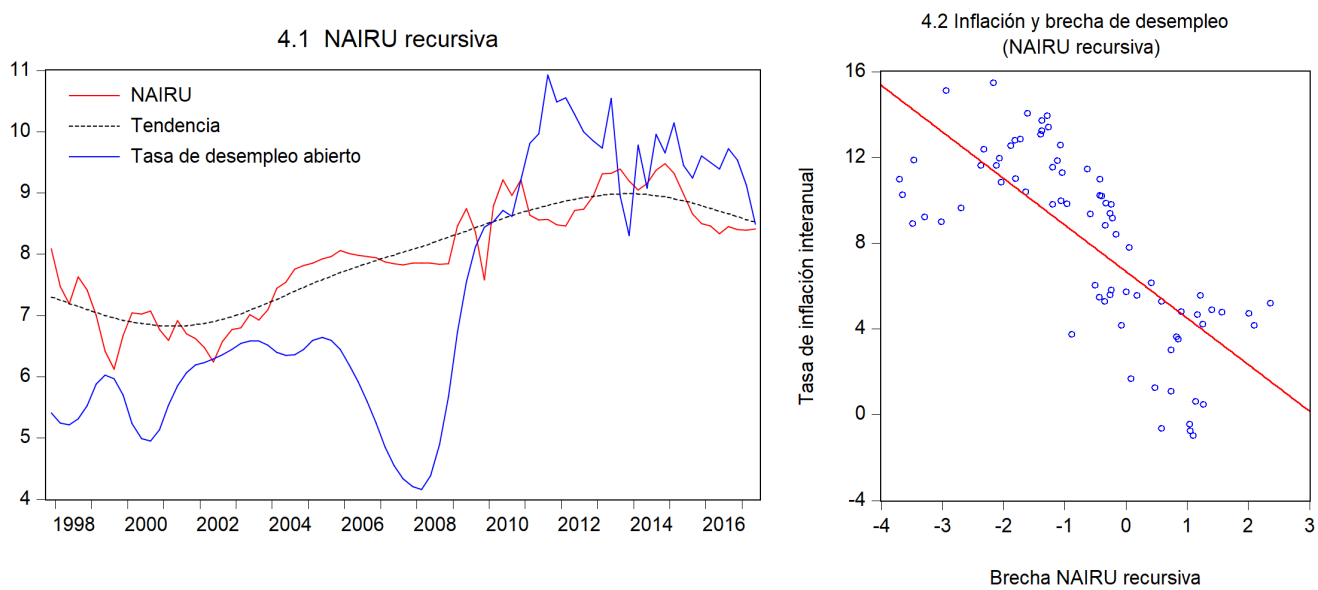

Fuente: Elaboración propia.

\section{NAIRU variable: Método de Ball y Mankiw}

Ball y Mankiw (2002) proponen un método simple para obtener una NAIRU que varía en el tiempo. Estos autores sugieren que la Curva de Phillips se puede reescribir de acuerdo con la ecuación (4):

$$
\Delta \pi_{t}=\beta U_{t}^{*}-\beta U_{t}+v_{t}
$$

17 Se siguió la misma metodología para calcular una NAIRU variable con base en la IMT; sin embargo, los resultados de esta estimación brindaron una NAIRU sumamente variable, la cual carece de sentido práctico por lo que los resultados no se presentan. 
Esos autores plantean que si se sabe el valor del parámetro $\beta$ en (5) entonces esta ecuación se puede expresar como sigue:

$$
U_{t}^{*}+v_{t} / \beta=U+\Delta \pi_{t} / \beta
$$

El lado derecho de la igualdad de la ecuación (5) se puede estimar a partir de los datos si se conoce el valor del parámetro $\beta$, lo cual da una estimación de $\left(U_{t}^{*}+v_{t} / \beta\right)$ donde $U_{t}^{*}$ representa la tendencia de largo plazo de la tasa de desempleo y $v_{t} / \beta$ es proporcional a los choques de oferta de corto plazo. Ball y Mankiw proponen extraer la NAIRU por medio del filtro de Hodrick-Prescott (Filtro HP) al lado derecho de (5) para hacer esta separación.

En esta estimación se usa el valor del parámetro $\beta$ obtenido de la ecuación (1), $\hat{\beta}=-0,25$ para la estimación que usa la inflación IPC y un valor de $-0,15$ para la estimación con base en la IMT. Por otra parte, se utiliza un valor de 1800 para el parámetro $\lambda$ del filtro HP, el cual penaliza la aceleración de la tendencia calculada por este filtro ${ }^{18}$. Adicionalmente, también se aplica el filtro HP directamente sobre la serie de tasa de desempleo para obtener una aproximación de la NAIRU. Las estimaciones así obtenidas se denominan NAIRU BMKIPC, NAIRU BMKIMT y NAIRU HP.

FIGURA 5

NAIRU ESTIMADA CON EL MÉTODO DE BALL Y MANKIW Y EL FILTRO HP
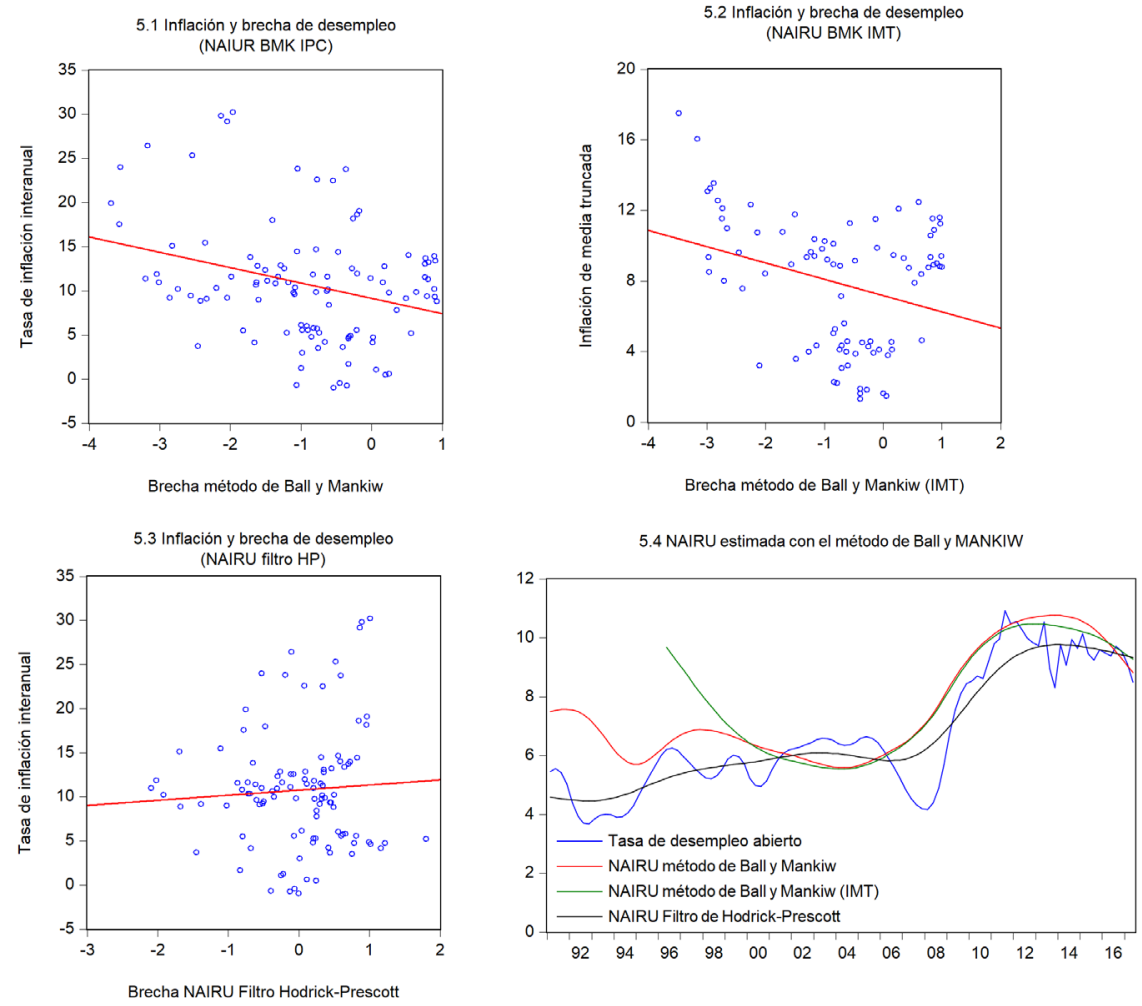

Fuente: Elaboración propia.

18 Conforme más alto es el valor de este parámetro, la tendencia estimada es más suave (menos volátil) y cuando este tiende al infinito, la tendencia converge a la que se estimaría mediante un modelo de tendencia temporal lineal. Cuando $\lambda$ se hace más pequeño, la tendencia estimada se parece a la serie original, al reducir las fluctuaciones del componente cíclico. 
La figura 5 muestra los resultados de estimar la NAIRU con el método de Ball y Mankiw $y$ con el filtro HP. Como se observa, la NAIRU estimada con la inflación IPC o IMT no difieren sustancialmente, a excepción de finales de los noventa, cuando la NAIRU BMKIMT exhibe niveles bastante mayores. Estas NAIRU muestran una brecha negativa la mayor parte del tiempo, a excepción del período comprendido entre los años 2001 y 2006 y un muy breve lapso entre los años 2011 y 2012. Al final de la muestra, ambas coinciden en señalar la existencia de una brecha de desempleo negativa, aunque en menor medida la estimada con la inflación IPC.

Por su parte, la NAIRU HP por su construcción, muestra períodos cíclicos relativamente cortos en los que la brecha de desempleo oscila entre positiva y negativa. Con este método, la brecha de desempleo calculada al segundo trimestre de 2017 es negativa, lo cual indica la presencia de presiones de demanda. No obstante, al contrario de las brechas estimadas con el método de Ball y Mankiw, la obtenida con la NAIRU HP está correlacionada positivamente con la inflación $(0,06)$ aunque esta correlación es débil. La correlación entre la brecha de desempleo calculada tanto con la inflación IPC como con la IMT es negativa y se estima en -0,3.

\section{NAIRU estimada con el filtro de Kalman}

Como se mencionó anteriormente, el filtro de Kalman ha sido tradicionalmente uno de los métodos más populares para obtener estimaciones de una NAIRU que varía en el tiempo. El filtro de Kalman es un algoritmo matemático utilizado para estimar sistemas dinámicos en representación estado-espacio. Una de sus ventajas es que permite obtener estimaciones e inferencias acerca del estado de variables no observables, tal como la NAIRU en este caso. El sistema de ecuaciones estimado es el siguiente ${ }^{19}$ :

$$
\begin{array}{rr}
\pi_{t}=\beta_{1} \pi_{t-1}+\beta_{2} \pi_{t}^{*}+\beta_{3}\left(U_{t-2}-U_{t}^{*}\right)+\varepsilon_{t} & \varepsilon_{t} \square N\left(0, \sigma_{\varepsilon}^{2}\right) \\
U_{t}^{*}=U_{t-1}^{*}+v_{t} & U_{t} \square N\left(0, \sigma_{v}^{2}\right)
\end{array}
$$

Donde la ecuación (6) es la Curva de Phillips, mientras que la ecuación (7) muestra el proceso que genera la NAIRU no observable, el cual supone que es un proceso estocástico que sigue un paseo aleatorio. Un aspecto importante para tomar en cuenta en la estimación de la NAIRU con este método tiene que ver con la razón de señal a ruido (signal-to-noise-ratio). Esta razón mide la volatilidad de la NAIRU en relación con la volatilidad de la inflación. Estimar el sistema de ecuaciones anterior sin restringir esta razón, por lo general da lugar a estimaciones de la NAIRU que son muy volátiles. Por otra parte, fijar esta razón en un valor igual a cero implicaría obtener una estimación de una NAIRU constante.

La selección de la razón de señal a ruido es altamente arbitraria y no existe una regla universalmente aceptada para su fijación. Sin embargo, en la literatura es usual experimentar con valores bajos de esta razón, en tanto se supone que la NAIRU evoluciona de forma relativamente lenta debido a que depende de factores estructurales que a su vez cambian de forma paulatina en el tiempo. 
Autores como Greenslade, Pierse y Salaheen (2003) usan un valor de 0,16 mientras que Gordon (1998) utiliza un valor de 0,2. En esta estimación, la razón de señal a ruido se calculó con base en una primera estimación de las ecuaciones (6) y (7) por mínimos cuadrados ordinarios ${ }^{20}$. Luego, la razón de señal a ruido se calculó dividiendo el error estándar de las ecuaciones, lo cual dio como resultado un valor de 0,25. En el caso del sistema estimado usando la IMT se decidió usar el mismo valor. Como se verá en breve, esto da como resultado estimaciones de NAIRU que varían en el tiempo con relativa suavidad, por lo que parece ser un valor apropiado. El cuadro 4 del Anexo I muestra las estimaciones de ambos modelos.

Los gráficos 6.1 y 6.2 de la figura 6 muestran las NAIRU estimadas con el filtro de Kalman. Las series presentadas corresponden a la serie suavizada, la cual utiliza toda la información en vez de la serie filtrada, que usa información hasta el momento en que el pronóstico se realizó. El área sombreada corresponde a un intervalo de confianza de \pm 1 error estándar, el cual en promedio es de 1 p.p. para la NAIRU estimada con la inflación IPC (en adelante NAIRU IPC) y de 0,7 p.p. para la estimada con la IMT (NAIRU IMT).

FIGURA 6

\section{NAIRU ESTIMADA CON EL FILTRO DE KALMAN}
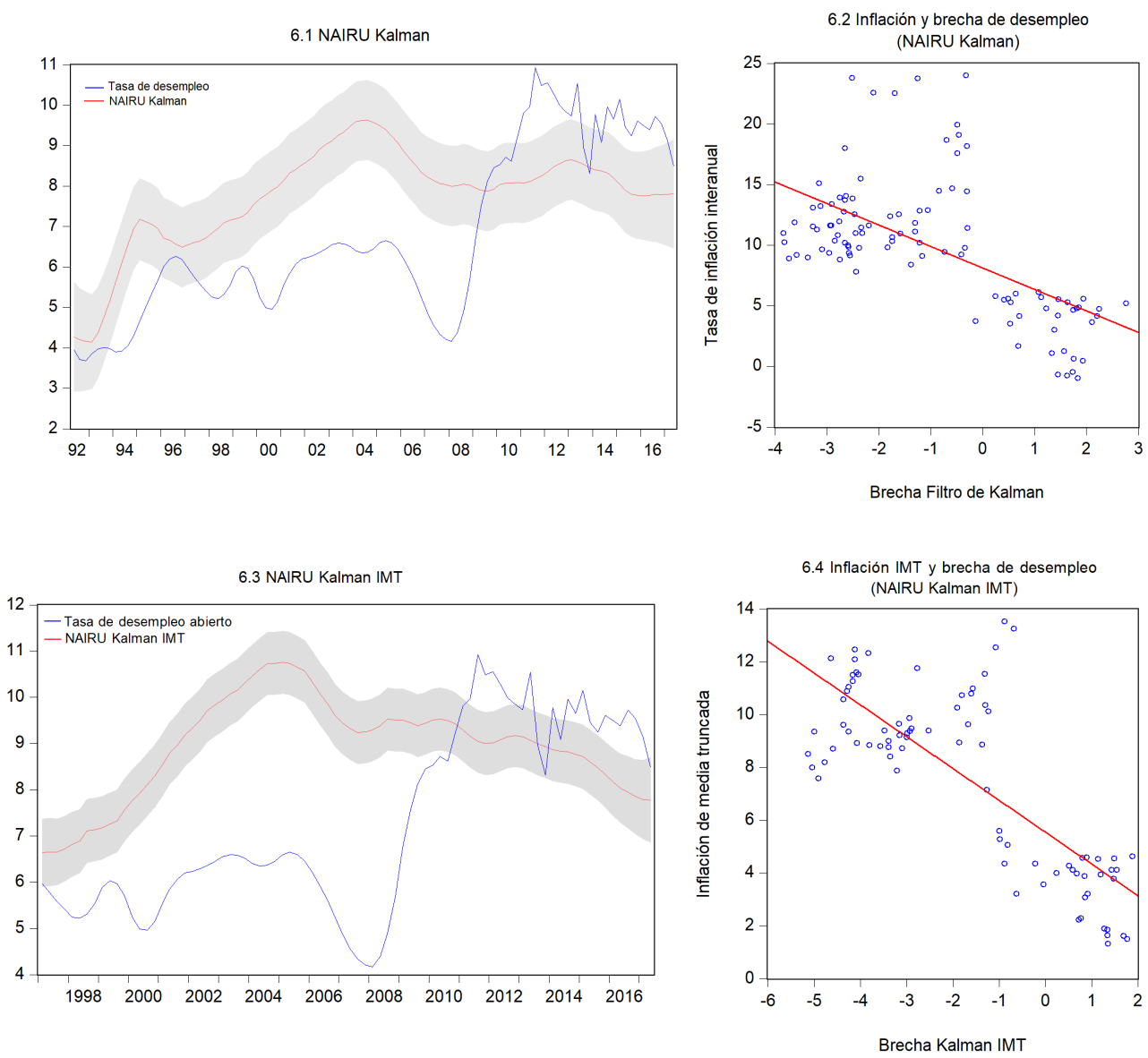

Fuente: Elaboración propia.

20 De esta estimación también se toman los valores iniciales de los parámetros del sistema. 
Como se puede apreciar, la estimación puntual de la NAIRU muestra una brecha negativa hasta el 2008, la cual se vuelve positiva a partir del 2009, exhibe un patrón muy similar al que se observa con las NAIRU estimadas con los otros métodos. Por su parte, la brecha de desempleo estimada con la NAIRU IMT se torna positiva hasta el año 2011, lo cual indica la ausencia de presiones de demanda agregada la mayor parte del tiempo.

A diferencia de las otras NAIRU que varían en el tiempo, estas series muestran una tendencia a reducirse entre los años 2005 y 2007, estabilizándose la NAIRU IPC a partir de ese año en un valor en torno al 8\%. En el caso de la NAIRU IMT, esta muestra una leve tendencia a reducirse a partir del año 2010. Al segundo trimestre de 2017, la NAIRU IPC se ubicaba en 8\%; mientras que la NAIRU IMT en 7,8\%. Además, dado el error estándar, en ambas estimaciones la tasa de desempleo observada no es estadísticamente distinta de la NAIRU, por lo que puede decirse que al final de la muestra la brecha de desempleo está cerrada. La correlación entre la brecha de desempleo calculada con la NAIRU IPC y la tasa de inflación es de -0,61, mientras que la correlación entre brecha de desempleo a partir de la NAIRU IMT y la IMT es de $-0,8$.

La figura 9 del Anexo I compara ambas estimaciones, donde destaca que, aunque siguen tendencias muy similares, la NAIRU IMT tiende a mostrar niveles algo mayores a partir del año 2000, alcanzándose la máxima diferencia entre ambas series en el primer trimestre de 2009 (1,52 p.p.).

\section{Evolución de la relación entre brecha de desempleo e inflación en Costa Rica}

Como se discutió en la sección II, en los últimos años se ha desarrollado un debate respecto a si la relación entre inflación y desempleo se ha debilitado. Además, en la sección IV, se evidenció como la relación entre inflación y desempleo en Costa Rica se ha manifestado con diferentes grados de intensidad dependiendo del período analizado.

No obstante, para efectos de explicar y pronosticar la inflación, conviene más estudiar si la brecha de desempleo (en vez de la tasa de desempleo) continúa manteniendo una asociación negativa con la tasa de inflación.

La figura 7 muestra la relación entre inflación y brecha de desempleo a partir del primer trimestre del 2010 y hasta el segundo trimestre de 2017, de acuerdo con los diferentes métodos utilizados. Destaca que la brecha de desempleo calculada con la NAIRU constante y la NAIRU HP está relacionada positivamente con la inflación.

Para estudiar más a fondo esta cuestión, se realiza una estimación recursiva de una Curva de Phillips en que las presiones de demanda son aproximadas por la brecha de desempleo. Dado que el interés está en analizar si esta relación ha cambiado en el tiempo, se realiza una estimación recursiva de la ecuación (7) por MCO en la que se deja fijo el final de la muestra y luego se elimina progresivamente una observación al inicio. La estimación final corresponde al periodo 2010:3 2017:2 de modo que incluye 28 observaciones. 
FIGURA 7

INFLACIÓN Y BRECHA DE DESEMPLEO: 2010:1 2017:2
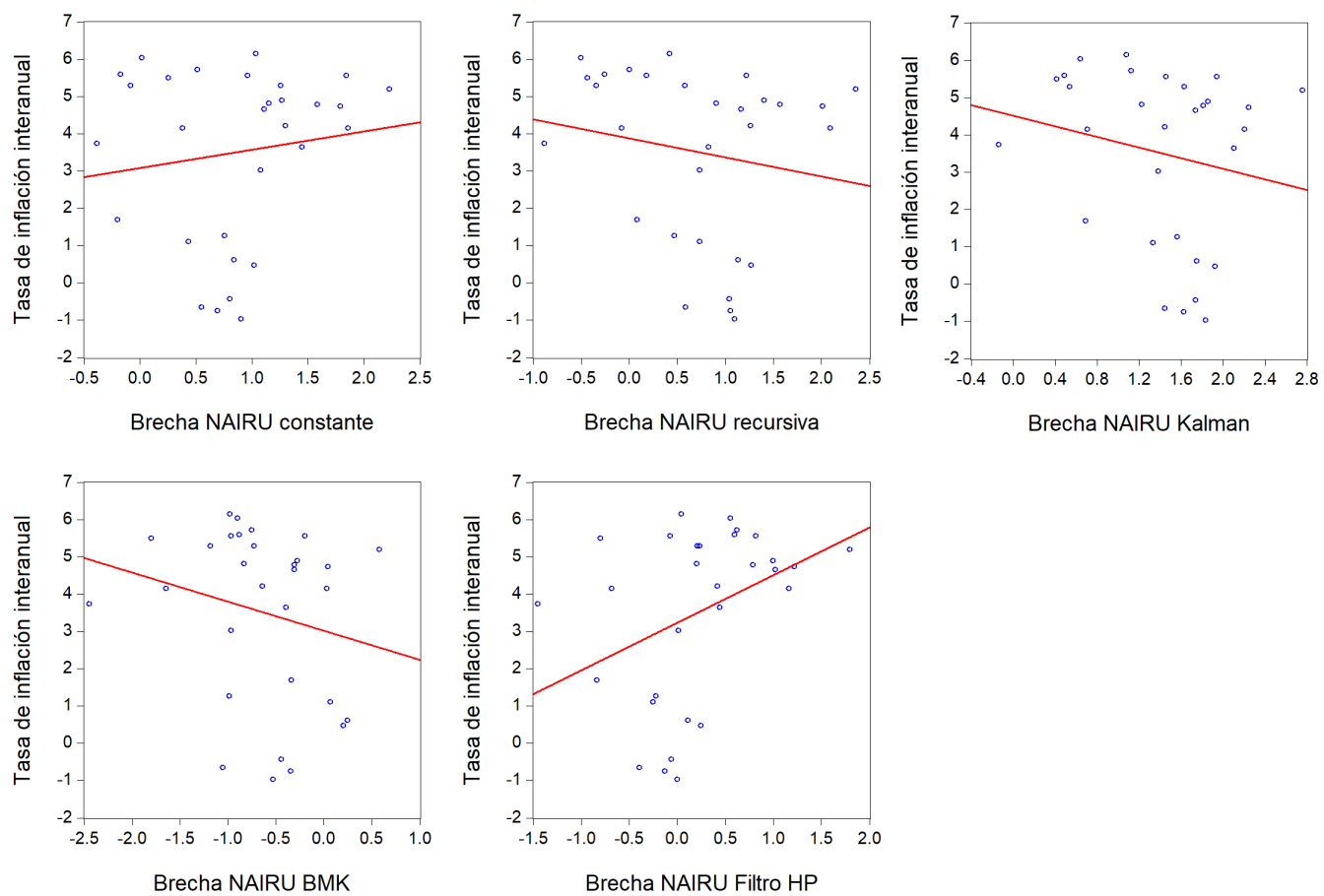

Fuente: Elaboración propia.

La figura 8 presenta la evolución en el tiempo del coeficiente que mide el efecto de la brecha de desempleo sobre la inflación. El panel izquierdo muestra la estimación puntual del coeficiente (línea azul), mientras que las líneas rojas punteadas corresponden a una banda de confianza de dos desviaciones estándar. El valor del coeficiente obtenido en cada estimación se ubica al inicio de la muestra.

El panel de la derecha exhibe el valor-p asociado a la hipótesis de que el coeficiente es igual a cero, mientras que la línea roja corresponde al nivel de significancia del 5\%. La figura 10 muestra el mismo análisis pero para las brechas que se estiman con base en la IMT. Como puede verse, las conclusiones que se obtienen respecto a la evolución de la relación entre brecha de desempleo e inflación, no difieren sustancialmente si se utilizan estas brechas. 


\section{EVOLUCIÓN DEL COEFICIENTE ASOCIADO A LA BRECHA DE DESEMPLEO EN LA CURVA DE PHILLIPS \\ FIGURA 8}

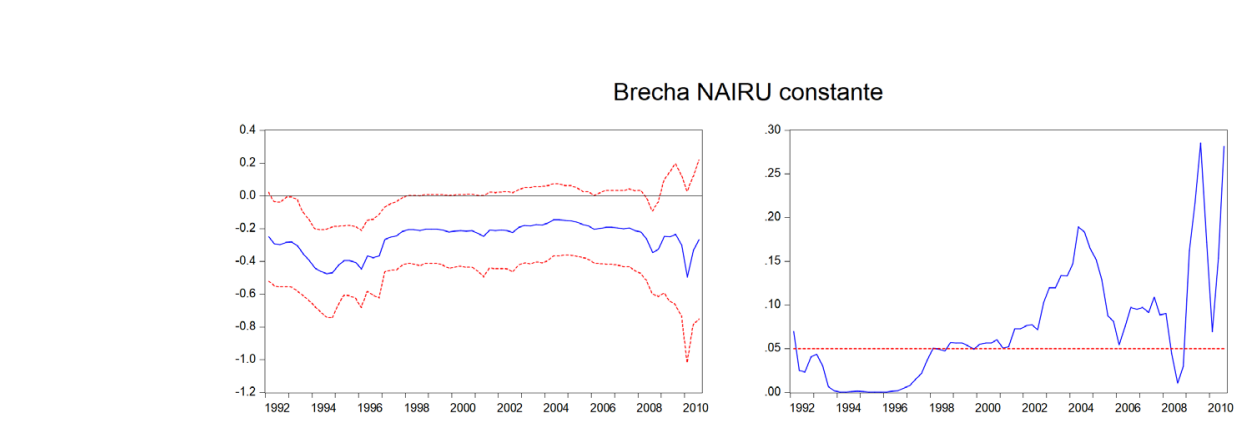

Brecha NAIRU recursiva
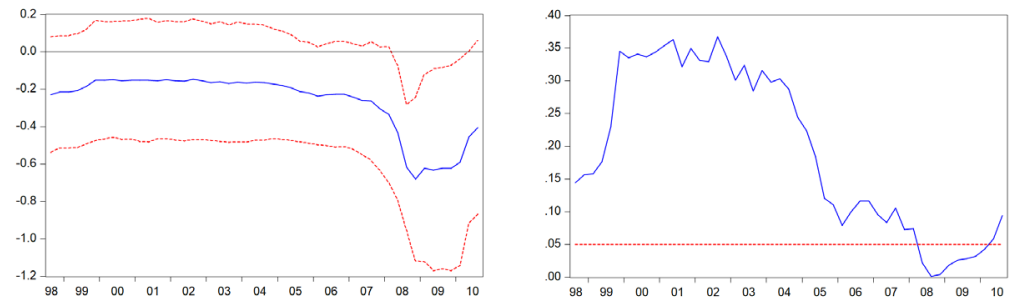

Brecha NAIRU HP
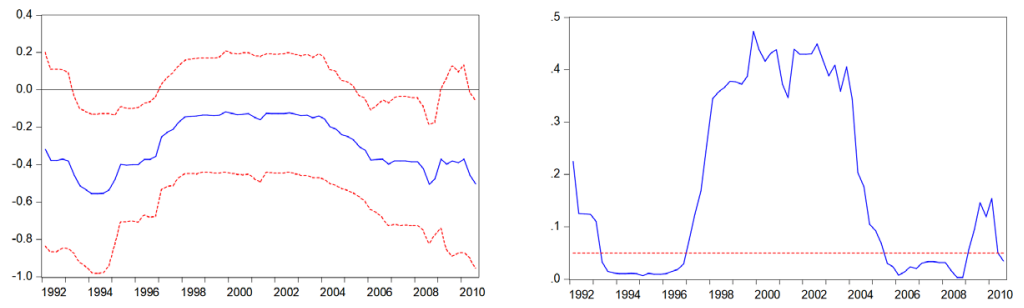

Brecha NAIRU BMK
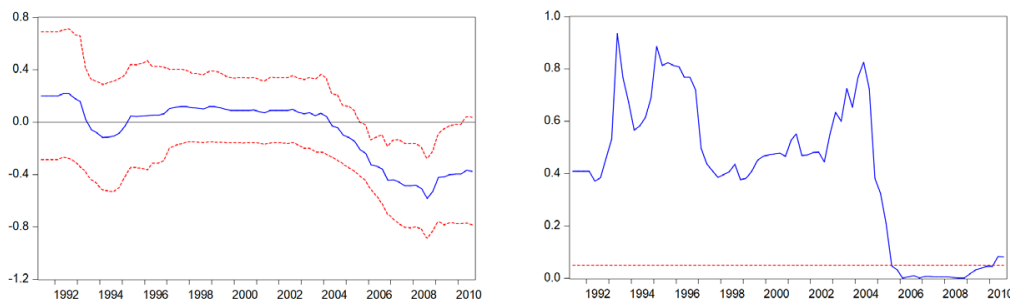

Brecha NAIRU Kalman
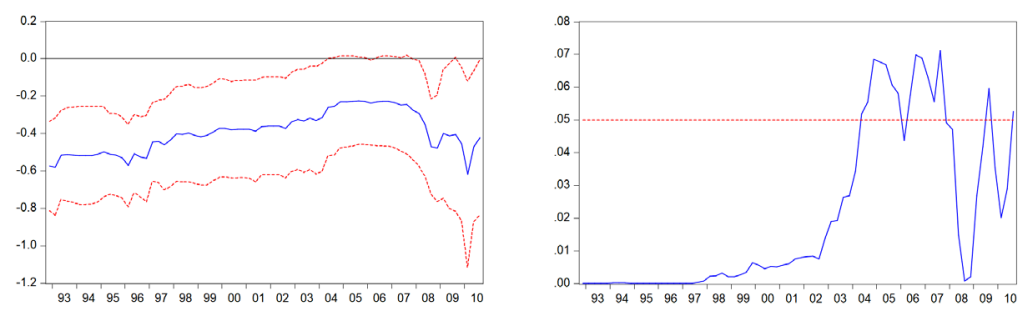

Fuente: Elaboración propia. 
Las brechas calculadas con la NAIRU constante, el filtro HP y el método de Ball y Mankiw exhiben un patrón similar al inicio de la década de los noventas, muestran una reducción del coeficiente asociado a la brecha de desempleo de alrededor de 0,2 p.p. entre 1992 y 1994. En el caso de la brecha NAIRU BMK, el coeficiente incluso pasa de positivo a negativo, aunque no es estadísticamente significativo, como sí lo son los coeficientes de las brechas NAIRU constante y NAIRU HP.

Luego, entre 1994 y 1996, este muestra una tendencia al aumento en conjunto con su significancia estadística ${ }^{21}$. A partir de este último año y hasta aproximadamente el 2004 muestran relativa estabilidad. En el caso de la brecha NAIRU recursiva esta se mantuvo estable un valor en torno a - 0,2 hasta el 2004. Mientras que el coeficiente asociado a la brecha NAIRU Kalman presenta una tendencia a incrementarse (hacerse menos negativo).

Para todas las brechas se observa que, aproximadamente luego del año 2004, el coeficiente de la brecha de desempleo muestra una tendencia a reducirse (hacerse más negativo), lo cual es indicativo de un fortalecimiento de la relación entre inflación y la brecha de desempleo.

Para las brechas Recursiva, HP y BMK, la reducción del coeficiente va acompañada de un aumento en su significancia estadística, ubicándose los valores-p del coeficiente para la estimación final (2010:3 2017:2) en 0,09, 0,03, y 0,08, respectivamente. Por su parte, la significancia estadística de las brechas de desempleo asociadas a la NAIRU constante y Kalman muestra un patrón contrario, evidencia de una fuerte desmejora que se acentúa a partir del año 2001. En el caso de la brecha NAIRU constante, luego de ese año el coeficiente deja de ser significativo a excepción de un breve período en el 2008. Para la brecha NAIRU Kalman, a pesar de que en general su significancia se reduce, esta continúa ubicándose por debajo del 10\% la mayor parte del tiempo.

En resumen, puede decirse que la relación entre brecha de desempleo e inflación se fortaleció por un breve periodo a inicios de los noventa. Luego, a partir de 1994, se deterioró y no fue estadísticamente significativa la mayor parte del tiempo entre 1994 y el 2004, aunque se mantuvo relativamente estable.

A partir de ese año se observa un fortalecimiento de la relación, volviéndose el coeficiente más negativo y en algunos casos mostrando una mayor significancia estadística. Por lo tanto, parece que la brecha de desempleo se puede utilizar como un indicador de las presiones de demanda agregada en Costa Rica.

En particular, las brechas NAIRU constante y Kalman pueden utilizarse en la elaboración de pronósticos de inflación, en tanto existe evidencia de causalidad en sentido de Granger hacia la inflación, tal y como lo muestra el cuadro 3, el cual presenta los valores-p de la prueba de causalidad de Granger para diferentes órdenes de rezagos ${ }^{22}$.

21 Es necesario acotar que conforme disminuye la cantidad de información con que se estiman los coeficientes, se reducen los grados de libertar y, por ende, la precisión con que son estimados. Por este motivo, es de esperar que el intervalo de confianza se ensanche o incremente conforme se utiliza una cantidad progresivamente menor de información.

22 Las conclusiones no cambian sustancialmente si se utilizan las brechas calculadas con la inflación IMT (ver cuadro 5 del anexo). En el caso de la brecha calculada con el filtro HP, hay evidencia de causalidad en ambos sentidos. 
CUADRO 3

PRUEBA DE CAUSALIDAD EN EL SENTIDO DE GRANGER

\begin{tabular}{lcccc}
\multicolumn{1}{c}{ HIPÓTESIS } & \multicolumn{3}{c}{ REZAGOS } \\
\cline { 2 - 5 } Ho: Inflación no causa a brecha NAIRU constante & 1 & 2 & 3 & 0,36 \\
Ha: Brecha NAIRU constante no causa a inflación & 0,58 & 0,37 & 0,31 & 0,01 \\
\hline Ho: Inflación no causa a brecha NAIRU recursiva & 0,77 & 0,01 & 0,01 & 0,44 \\
Ha: Brecha NAIRU recursiva no causa a inflación & 0,27 & 0,27 & 0,44 & 0,27 \\
\hline Ho: Inflación no causa a brecha NAIRU ByM & 0,24 & 0,20 & 0,28 & 0,38 \\
Ha: Brecha NAIRU BMK no causa a inflación & 0,47 & 0,91 & 0,89 & 0,92 \\
\hline Ho: Inflación no causa a brecha NAIRU Kalman & 0,13 & 0,31 & 0,32 & 0,15 \\
Ha: Brecha NAIRU Kalman no causa a inflación & 0,00 & 0,01 & 0,01 & 0,00 \\
\hline
\end{tabular}

Fuente: Elaboración propia.

\section{CONCLUSIONES}

En este documento se presentan varias estimaciones de la tasa de desempleo no aceleradora de la inflación o NAIRU para Costa Rica. Una primera estimación a partir de una Curva de Phillips brinda una estimación constante de la NAIRU, ubicándola en $8,7 \%$. No obstante, un intervalo de confianza al $95 \%$ calculado con base en el método gaussiano propuesto por Staiger, Stock y Watson (1996) sugiere que la NAIRU se estima con bastante imprecisión, en tanto el intervalo la ubica en un rango comprendido entre 3,9 y $13,1 \%$.

Dado que se ha sugerido que la NAIRU no es constante, sino que puede variar en el tiempo, esta se aproxima por otros métodos con el fin de obtener un indicador variable. En particular, se obtienen NAIRU variables con base en una estimación recursiva de la Curva de Phillips, el método propuesto por Ball y Mankiw (2002), el filtro de Hodrick y Prescott y el filtro de Kalman.

Estas estimaciones indican que la NAIRU se ha incrementado en Costa Rica en relación con los niveles observados a inicios de los años noventa, aumento que se ha acentuado principalmente luego del año 2008. Solamente la NAIRU calculada con el filtro de Kalman muestra un patrón distinto, reduciéndose a partir del año 2004. Por otra parte, las NAIRU variables que se calculan con base en la IMT muestran un patrón que no difiere sustancialmente del observado en las estimaciones realizadas con base en la inflación del IPC.

Las brechas de desempleo calculadas con base en las distintas NAIRU ( $U_{-} t-U_{-}{ }^{\wedge *}$ ) están relacionadas negativamente con la tasa de inflación a excepción de la que se obtiene con el filtro HP. Además, la evolución de estas brechas coincide en señalar que esta tendió a ser negativa durante los noventa y la primera mitad de la década pasada. A partir de ese momento, ha sido mayormente positiva, lo cual es indicativo de ausencia de presiones de demanda agregada, lo que además coincide con un período en que la inflación se ha mantenido en niveles de un dígito, históricamente bajos. Para el segundo trimestre de 2017, las NAIRU calculadas indican que las brechas de desempleo estaban cerradas.

Dada una coyuntura internacional en la que se pone en duda si la relación entre inflación y desempleo continúa siendo válida, se realiza una estimación recursiva del coeficiente asociado con la brecha de desempleo en la Curva de Phillips con el fin de determinar si esta variable continúa resultando efectiva para explicar la inflación en Costa Rica. El resultado de este análisis sugiere que, en el período en estudio, esta relación se ha manifestado con diferentes grados de intensidad. No obstante, parece que a partir del año 2004 se da un fortalecimiento de la relación entre inflación 
y brecha de desempleo, en tanto el coeficiente tiende a volverse más negativo y en algunos casos, muestra una mayor significancia estadística. Por otra parte, de las brechas de desempleo calculadas, solamente las que se generan a partir de la NAIRU constante y la NAIRU estimada con el filtro de Kalman pueden utilizarse con fines de pronóstico de la inflación, dado que son las únicas para las cuales existe evidencia de causalidad en sentido de Granger.

\section{REFERENCIAS}

Ball, L., \& Mankiw, N. G. (2002). The NAIRU in Theory and Practice (NBER Working Paper No. 8940). https://doi.org/10.3386/w8940

Ball, L., \& Mazumder, S. (2011, spring). Inflation Dynamics and the Great Recession. Brookings Papers on Economic Activity. 42(1), 337-405. Recuperado de https://www.brookings.edu/ bpea-articles/the-evolution-of-inflation-dynamics-and-the-great-recession/

Banco Central de Costa Rica. (2015). Indices de Precios. Recuperado de: https://www.bccr.fi.cr/seccion-indicadores-economicos/\%c3\%adndices-de-precios

Blanchard, O. (2016). The US Phillips Curve: Back to the 60s. (Peterson Institute for International Economics Policy Brief No. PB16-1). Recuperado de https://www.piie.com/publications/policy-briefs/us-phillips-curve-back-60s

Blanchard, O., Cerutti, E., \& Summers, L. (2015). Inflation and Activity - Two Explorations and their Monetary Policy Implications (NBER Working Paper No. 21726). https://doi.org/10.3386/w21726

Boone, L., Giorno, C., Meacci, M., Rae, D., Richardson, P., \& Turner, D. (2003). Estimating the structural rate of unemployment for the OECD countries. OECD Economic Studies, 2001(2), 171-216. https://doi.org/10.1787/eco_studies-v2001-art14-en

Cheung, L., Leung, F. \& Lo, P. (2017). Understanding US Inflation Dynamics. (Hong Kong Monetary Authority. Research Memorandum 02/2017), China: Hong Kong Monetary Authority.

Coibion, O., \& Gorodnichenko, Y. (2015). Is the Phillips Curve Alive and Well after All? Inflation Expectations and the Missing Disinflation. American Economic Journal: Macroeconomics, 7(1), 197-232. https://doi.org/10.1257/mac.20130306

Daly, M. C., \& Hobijn, B. (2014). Downward Nominal Wage Rigidities Bend the Phillips Curve (Federal Reserve Bank of San Francisco Working Paper No. 2013-08). https://doi.org/10.24148/wp2013-08

Denton, F. T. (1971). Adjustment of Monthly or Quarterly Series to Annual Totals: An Approach Based on Quadratic Minimization. Journal of the American Statistical Association, 66(333), 99-102. https://doi.org/10.1080/01621459.1971.10482227

Estrella, A., \& Mishkin, F. (2000). Rethinking the Role of NAIRU in Monetary Policy: Implications of Model Formulation and Uncertainty (NBER Working Paper No. 6518). https://doi.org/10.3386/w6518

Fabiani, S., \& Mestre, R. (2000). Alternative measures of the NAIRU in the euro area: Estimates and assessment (European Central Bank Working Paper Series No. 17).

Gordon, R. (1996). The Time-Varying NAIRU and its Implications for Economic Policy (NBER Working Paper No. 5735). https://doi.org/10.3386/w5735

Greenslade, J., Pierse, R., \& Saleheen, J. (2003). A Kalman filter approach to estimating the UK NAIRU. (Bank of England Working Paper No. 179). Londres: Bank of England.

Instituto Nacional de Estadística y Censos (2000-2009). Encuesta de Hogares de Propósitos Múltiples [Base de datos]. San José, Costa Rica. Recuperado de: http://www.inec.go.cr/sistema-de-consultas

Instituto Nacional de Estadística y Censos (2010-2019a). Encuesta Nacional a Hogares. [Base de datos]. San José, Costa Rica. Recuperado de: 
http://www.inec.go.cr/sistema-de-consultas

Instituto Nacional de Estadística y Censos (2010-2019b). Encuesta Continua de Empleo. [Base de datos]. San José, Costa Rica. Recuperado de http://www.inec.go.cr/sistema-de-consultas

Instituto Nacional de Estadística y Censos (2019c). Índice de Precios al Consumidor. San José, Costa Rica. Recuperado de http://inec.cr/economia/indice-de-precios-al-consumidor

Kiley, M. T. (2015). An evaluation of the inflationary pressure associated with short- and long-term unemployment. Economics Letters, 137, 5-9. https://doi.org/10.1016/j.econlet.2015.10.005

Kiley, M. T. (2015). Low Inflation in the United States: A Summary of Recent Research. FEDS Notes, 2015(1665). https://doi.org/10.17016/2380-7172.1665

Krugman, P. (4 de diciembre de 2015). Anchors Away (Slightly Wonkish). New York Times blog. Recuperado de https://krugman.blogs.nytimes.com/2015/12/04/anchors-away-slightly-wonkish/

Laubach, T., \& Williams, J. C. (2003). Measuring the Natural Rate of Interest. Review of Economics and Statistics, 85(4), 1063-1070. https://doi.org/10.1162/003465303772815934

Modigliani, F., \& Papademos, L. (1975). Targets for Monetary Policy in the Coming Year. Brookings Papers on Economic Activity, 1975(1), 141-165. https://doi.org/10.2307/2534063

Richardson, P., Boone, L., Giorno, C., Meacci, M., Rae, D. \& Turner, D. (2000). The Concept, Policy Use and Measurement of Structural Unemployment: Estimating a Time Varying NAIRU Across 21 OECD Countries. (OECD Economics Department Working Paper No. 250). https://doi.org/10.1787/785730283515

Segura, C. \& Vindas, A. (2012). Estimación de la tasa de interés real neutral y la tasa natural de desempleo para la economía costarricense. (Documento de Investigación DEC-DIEDI-007-2012). Departamento de Investigación Económica. Banco Central de Costa Rica. Recuperado de: http://www.secmca.org/recard/index.php/foro/article/view/10

Staiger, D., Stock, J., \& Watson, M. (1996). How Precise are Estimates of the Natural Rate of Unemployment? (NBER Working Paper No. 5477). https://doi.org/10.3386/w5477

Stock, J. (2011). Discussion of Ball and Mazumder "Inflation Dynamics and the Great Recession". Brookings Papers on Economic Activity, 387-402. Recuperado de: https://scholar.harvard. edu/stock/publications/discussion-ball-and-mazumder-inflation-dynamics-and-greatrecession

Szeto, K. L., \& Guy, M. (2004). Estimating a New Zealand NAIRU. (Treasury Working Paper Series 04/10), New Zealand Treasury. Recuperado de: https://treasury.govt.nz/publications/wp/ estimating-new-zealand-nairu-wp-04-10-html

Torres, C. (2012). Costa Rica: Determinación de cambios estructurales en el nivel de la tasa de inflación: periodo 1997-2011. (Documentos de Trabajo No. 02-2012). Departamento de Investigación Económica. División Económica. Banco Central de Costa Rica. Recuperado de: https://activos.bccr.fi.cr/sitios/bccr/investigacioneseconomicas/DocPoliticaMonetariaInflacion/ DT-02-2012_Determinacion_cambios_estructurales_inflacion1997-2011.pdf 
ANEXO 1. CUADROS Y GRÁFICOS

CUADRO 4

ESTIMACIÓN DE LOS MODELOS ESTADO-ESPACIO CON EL FILTRO DE KALMAN

\begin{tabular}{ccccc}
\multirow{2}{*}{ VARIABLE } & \multicolumn{2}{c}{ INFLACIÓN IPC } & \multicolumn{2}{c}{ IMT } \\
\cline { 2 - 5 } & Coeficiente & Valor-P & Coeficiente & Valor-P \\
$\pi_{t-1}$ & $-0,20$ & 0,89 & 0,07 & 0,92 \\
$\pi_{t-2}$ & 0,73 & 0,00 & 0,94 & 0,00 \\
$e_{t}$ & & & $-0,20$ & 0,15 \\
$\pi_{t}^{*}$ & 0,58 & 0,01 & 0,05 & 0,02 \\
$U_{t}-U_{t-2}^{*}$ & $-0,42$ & 0,02 & 0,42 & 0,00 \\
\hline
\end{tabular}

Fuente: Elaboración propia.

FIGURA 9

NAIRU ESTIMADA CON EL FILTRO DE KALMAN

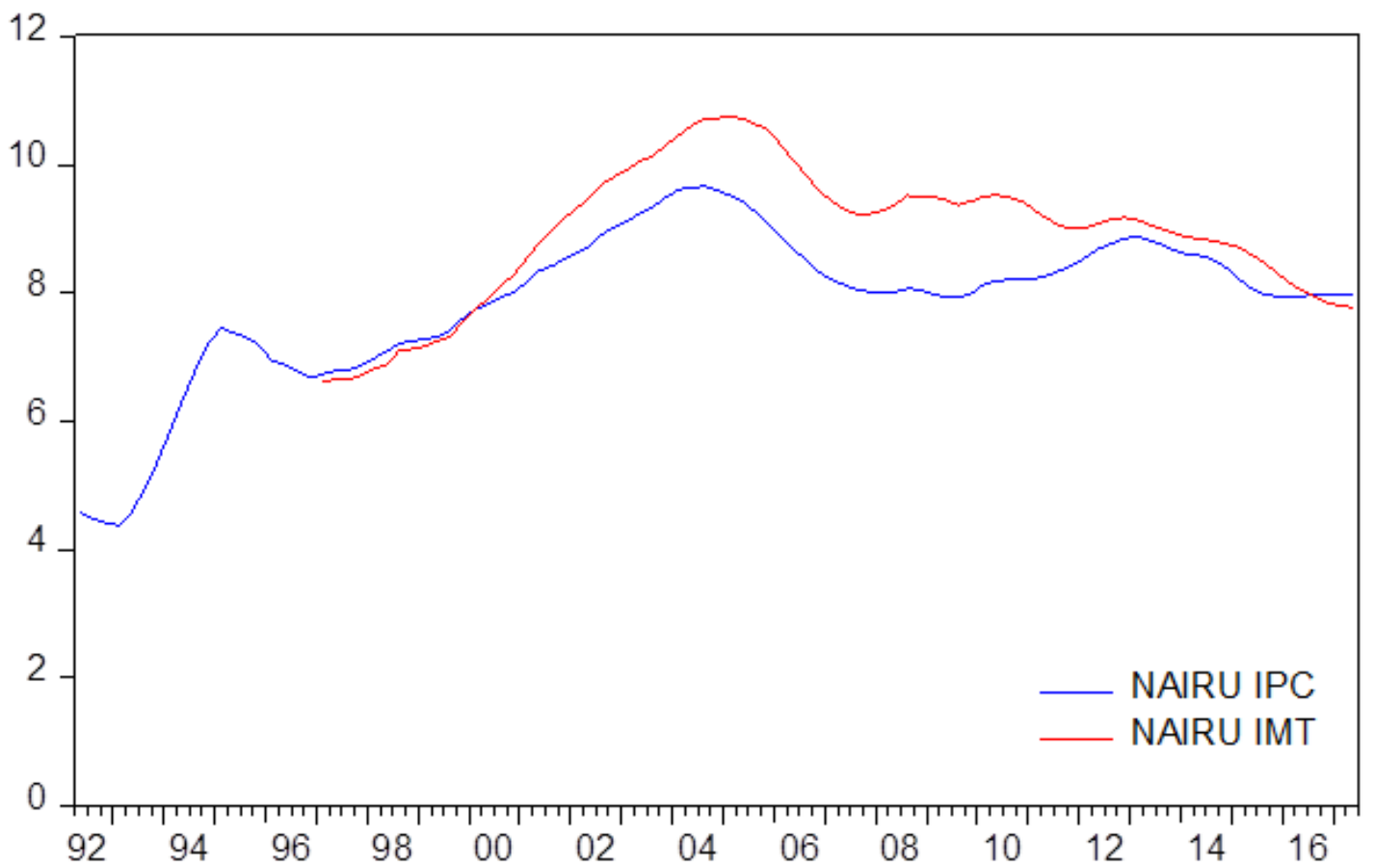

Fuente: Elaboración propia. 
FIGURA 10

EVOLUCIÓN DEL COEFICIENTE ASOCIADO A LA BRECHA DE DESEMPLEO EN LA CURVA DE PHILLIPS (IMT)

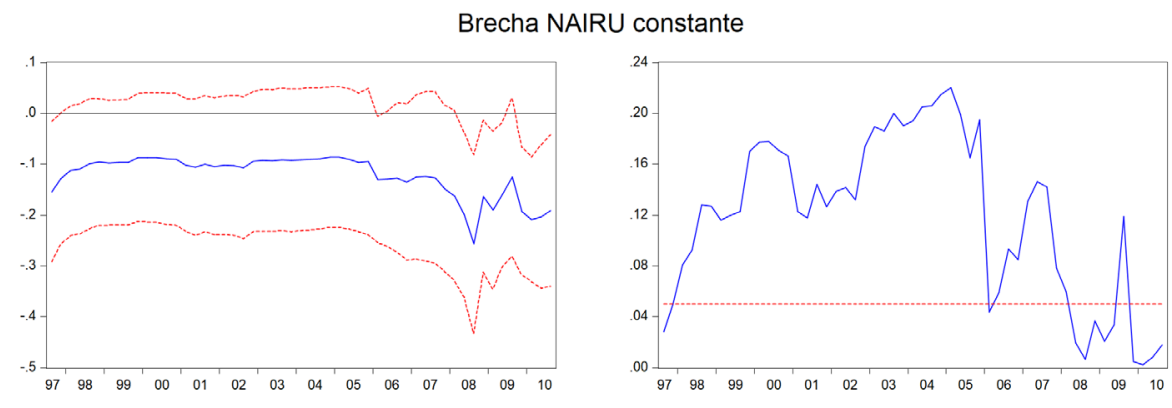

Brecha NAIRU BMK
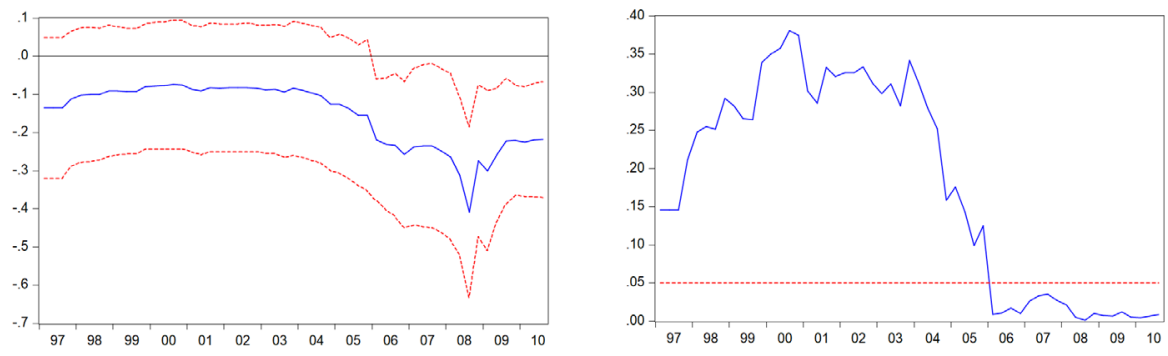

Brecha NAIRU filtro HP
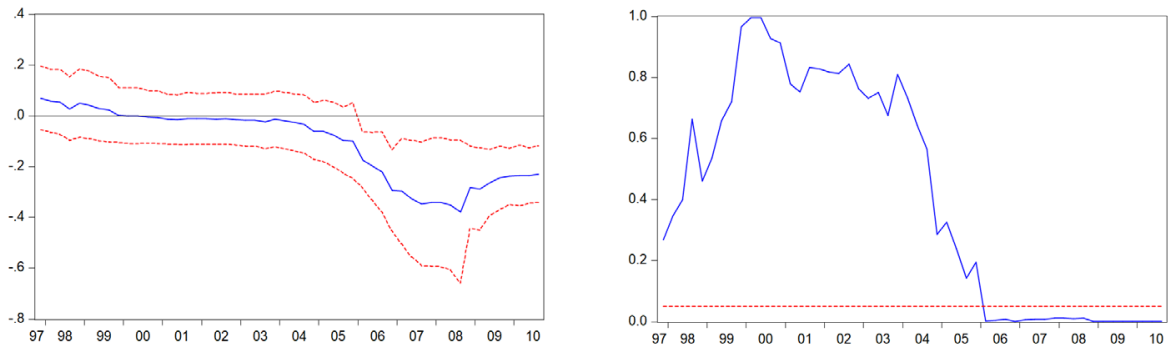

Brecha filtro de Kalman
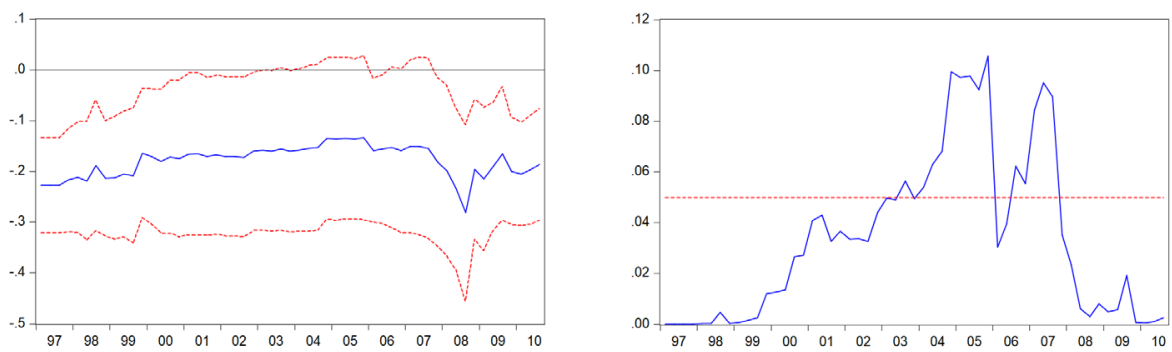

Fuente: Elaboración propia. 


\section{CUADRO 5 \\ PRUEBA DE CAUSALIDAD DE GRANGER \\ (INFLACIÓN IMT)}

\begin{tabular}{|c|c|c|c|c|}
\hline \multirow{2}{*}{ HIPÓTESIS } & \multicolumn{4}{|c|}{ REZAGOS } \\
\hline & 1 & 2 & 3 & 4 \\
\hline Ho: Inflación IMT no causa a brecha NAIRU constante & 0,80 & 0,89 & 0,72 & 0,55 \\
\hline $\begin{array}{l}\text { Ha: Brecha NAIRU constante no causa a inflación } \\
\text { IMT }\end{array}$ & 0,00 & 0,01 & 0,01 & 0,02 \\
\hline Ho: Inflación IMT no causa a brecha NAIRU ByM & 0,82 & 0,80 & 0,80 & 0,75 \\
\hline Ha: Brecha NAIRU BMK no causa a inflación IMT & 0,64 & 0,96 & 0,24 & 0,28 \\
\hline Ho: Inflación IMT no causa a brecha NAIRU filtro HP & 0,19 & 0,01 & 0,03 & 0,00 \\
\hline Ha: Brecha NAIRU filtro HP no causa a inflación IMT & 0,06 & 0,27 & 0,05 & 0,03 \\
\hline Ho: Inflación IMT no causa a brecha NAIRU Kalman & 0,72 & 0,92 & 0,85 & 0,84 \\
\hline Ha: Brecha NAIRU Kalman no causa a inflación IMT & 0,00 & 0,00 & 0,00 & 0,01 \\
\hline
\end{tabular}

Fuente: Elaboración propia. 